\title{
Design study for medium energy high intensity proton accelerator
}

\author{
Abhishek Pathak $\odot,{ }^{2}$ Shweta Roy, ${ }^{1}$ SVLS Rao, ${ }^{1}$ and Srinivas Krishnagopal ${ }^{1,3}$ \\ ${ }^{1}$ Ion Accelerator Development Division, Bhabha Atomic Research Centre, Mumbai, India \\ ${ }^{2} U M-D A E$ Centre for Excellence in Basic Sciences, Mumbai, India \\ ${ }^{3}$ Homi Bhabha National Institute, Mumbai, India
}

(Received 20 September 2019; accepted 31 August 2020; published 22 September 2020)

\begin{abstract}
A $200 \mathrm{MeV}, 10 \mathrm{~mA}$, medium energy high intensity proton accelerator (MEHIPA) is proposed to form the second stage of the Indian accelerator-driven system program. The linac consists of a normal conducting $3 \mathrm{MeV}$ radio frequency quadrupole (RFQ) and a $200 \mathrm{MeV}$ superconducting section consisting of three different families of single spoke resonators (SSR-A, SSR-B, and SSR-C). Here we discuss a detailed physics design of MEHIPA, including electro-magnetic design, multipacting analysis, higher-order mode studies, and beam dynamics. We have designed a compact linac, by careful optimization of cavity $\beta$ for the superconducting section. We also performed detailed optimization studies on the linac lattice and transport line parameters, aimed at minimizing the emittance and beam halo. Reliability analysis and error studies were performed on this optimized linac, including various beam, cavity, and magnet parameters, to estimate the operation tolerances, and the results are reported for a maximum emittance growth of $50 \%$.
\end{abstract}

DOI: 10.1103/PhysRevAccelBeams.23.090101

\section{INTRODUCTION}

Recent progress in accelerator technology has made it possible to use a proton accelerator to produce nuclear energy. In an accelerator-driven system (ADS), a highintensity proton accelerator is used to produce protons of around $1 \mathrm{GeV}$ energy, which strike a target such as lead or tungsten to produce spallation neutrons. These neutrons enter a subcritical core and induce nuclear reactions, including fission. Such a system is intrinsically safe because if the accelerator is turned off, the fission chains are self-terminating. ADS can be used to produce power, incinerate minor actinides and long-lived fission products, and for the utilization of thorium as an alternative nuclear fuel. The accelerator for ADS has to produce high energy $(1 \mathrm{GeV})$ protons and deliver tens of milliamperes of beam current with minimum $(<1 \mathrm{nA} / \mathrm{m})$ beam loss for hands-on maintenance of the accelerator. This makes the development of accelerators for ADS very challenging. Major efforts are on in this field in India [1], Europe [2], Japan [3], China [4], Korea [5], etc. In India, it was planned to take a staged approach toward the development of the requisite accelerator technology, and an ADS road-map (Fig. 1) was prepared in three phases: $20 \mathrm{MeV}, 200 \mathrm{MeV}$ [6], and $1 \mathrm{GeV}$. One of the most challenging parts of such a $\mathrm{CW}$ proton

Published by the American Physical Society under the terms of the Creative Commons Attribution 4.0 International license. Further distribution of this work must maintain attribution to the author(s) and the published article's title, journal citation, and DOI. accelerator is the development of the low-energy injector section because the space charge effects are maximum at these energies. With this in mind, the development of a low energy high-intensity proton accelerator (LEHIPA), as the front-end injector of the $1 \mathrm{GeV}$ accelerator for the ADS programme, was taken up at BARC, Mumbai [1,7-9]. After ascending a steep learning and technological curve, first beam acceleration (to $3 \mathrm{MeV}$ in the $352 \mathrm{MHz}$, LEHIPA RFQ) has already been demonstrated.

A $200 \mathrm{MeV}, 10 \mathrm{~mA}$, medium energy high-intensity proton accelerator (MEHIPA) is proposed to form the second stage of the Indian ADS program. The MEHIPA accelerator is one of the few CW, high energy accelerators with such high current (five-time higher than PIP-II, Fermilab [10]). MEHIPA will have a front-end similar to LEHIPA, i.e., a $50 \mathrm{keV}$ ECR ion source and a $3 \mathrm{MeV}$, $325 \mathrm{MHz}$ RFQ, but will go superconducting immediately after. For these medium energies, the accelerating structure of choice is the superconducting spoke resonator (SSR). The decision of $200 \mathrm{MeV}$ as the maximum energy of MEHIPA is predicated on two factors: (1) SSRs are viable and efficient accelerating structures in the intermediate energy range, and (2) at $200 \mathrm{MeV}$ there will be sufficient spallation reactions so that one can conceive coupling of the MEHIPA beam with a target and reactor system, in a Demo ADS Facility to test and validate the ADS concept. This paper discusses the physics design and optimization studies performed for the MEHIPA linac. The normal conducting injector section is addressed in Sec. II, where we detail the optimization of the LEBT channel, involving the drift length, solenoid fields and focusing scheme, to reduce emittance and halo blow-up while transporting the 


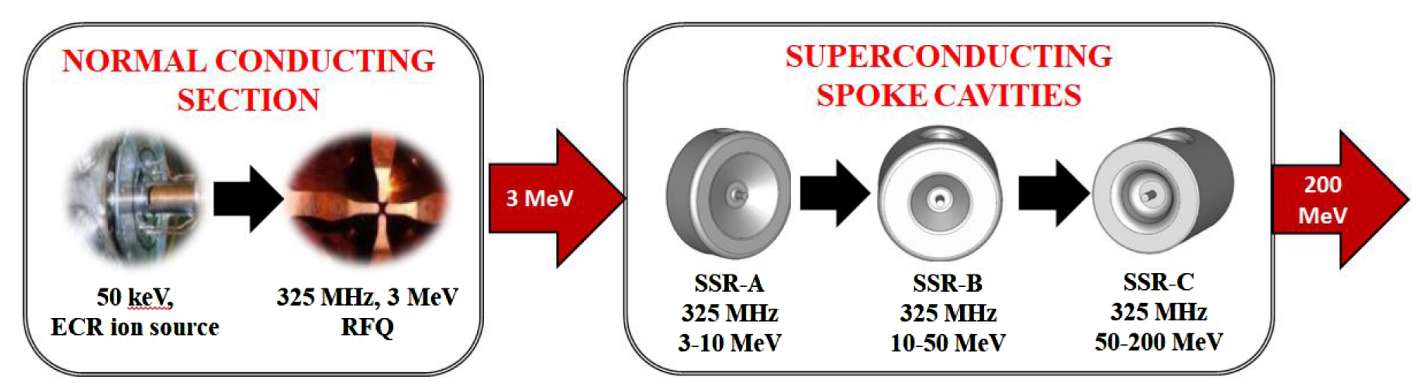

FIG. 1. Layout for the $200 \mathrm{MeV}$ medium energy high intensity proton accelerator (MEHIPA).

beam to the RFQ. This section also describes the beam dynamics simulations for the LEBT channel, and RFQ performed to investigate the dependence of beam characteristics on space-charge non-linearity and beam intensity. Section III discusses the beam dynamics during the transition from the normal conducting to the superconducting section of the linac using an optimized (quadrupole gradients and gap fields) medium energy beam transport (MEBT) line. The rf cavity design, peak fields optimization, calculation of optimal $\beta$, multipacting, and higherorder mode analysis for all three families (SSR-A, SSR-B, and SSR-C) of the spoke resonators are presented in Sec. IV of the paper. The 3D rf field from the optimized spoke cavities, and superconducting solenoids were used to perform beam dynamics simulations for the MEHIPA linac with a primary focus on minimization of emittance and beam halo growth by avoiding collective beam instabilities and parametric resonances in the lattice; the results of these simulations are discussed in Sec. V. In Sec. VI of this paper, we provide the details of the power requirements for the accelerating cavities in the linac. This section also gives the static and dynamic heat load for the SSR-A, SSR-B, and SSR-C cavities and cryomodules, forming the superconducting (SC) section of MEHIPA. As these accelerators are very sensitive to different kind of reliability and errors issues like beamline element failure and/or misalignments, component rotation, fabrication errors, magnet, and cavity field error, etc., we performed detailed reliability and statistical error analysis for MEHIPA linac to provide a quantitative estimate of tolerances for the linac, and the results are summarized in Sec. VII and Sec. VIII respectively.

\section{NORMAL CONDUCTING INJECTOR SECTION}

\section{A. LEBT channel}

As shown in Fig. 1, a $10 \mathrm{~mA}, 50 \mathrm{keV}$, axisymmetric proton beam from an ECR ion source was transported and matched to a $325 \mathrm{MHz}, 3 \mathrm{MeV}$ radio frequency quadrupole (RFQ) using a low energy beam transport (LEBT) channel. The proposed LEBT for MEHIPA [6] consists of two normal conducting solenoids to perform the transverse matching of the Courant-Snyder parameters [11]. The

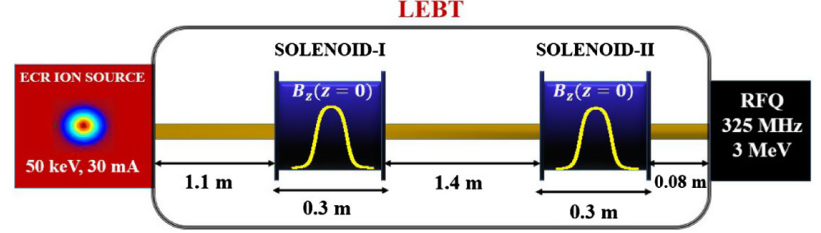

FIG. 2. Layout for the low energy beam transport channel for the MEHIPA linac.

schematic of the MEHIPA LEBT is shown in Fig. 2 and the beam parameters at the LEBT input are listed in Table I.

The magnetic field of the two solenoids was tuned independently to achieve the acceptance Twiss parameters at the RFQ input while keeping a check on the transverse emittance growth and halo [12] development. The matching was performed using the code TraceWin [13] with the matching criterion defined as,

$$
M F=\sqrt{1+\frac{\Delta+\sqrt{\Delta(\Delta+4)}}{2}},
$$

where $\Delta=(\Delta \alpha)^{2}-\Delta \beta \Delta \gamma$, corresponds to the difference between the matched beam Twiss parameters and actual Twiss parameters. The rms emittance and beam halo patameter is defined as,

TABLE I. Input beam and space-charge compensation parameters for the LEBT channel for a given acceptance of the RFQ.

\begin{tabular}{lc}
\hline \hline Parameter & Value \\
\hline Beam Current & $10 \mathrm{~mA}$ \\
Particle type & Proton \\
Particle energy & $50 \mathrm{keV}$ \\
Emittance & $0.2 \pi \mathrm{mm}-\mathrm{mrad}$ \\
Input $\alpha_{x, y}$ & -1.8 \\
Input $\beta_{x, y}$ & $0.24 \mathrm{~mm} / \pi \mathrm{mrad}$ \\
Output $\alpha_{x, y}$ & 1.56 \\
Output $\beta_{x, y}$ & $0.044 \mathrm{~mm} / \pi \mathrm{mrad}$ \\
Space-charge compensation & $95 \%$ \\
Input distribution & Gaussian, Parabolic, \\
& $4 \mathrm{D}$ water bag, KV \\
Number of macro-particles & $5 \times 10^{6}$ \\
\hline \hline
\end{tabular}



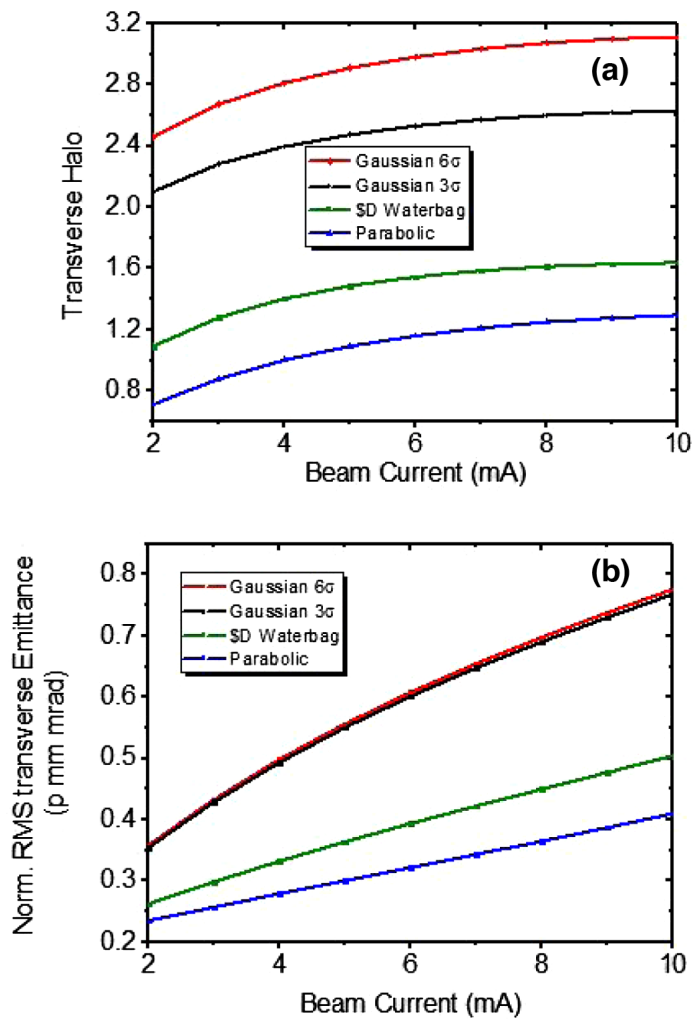

FIG. 3. Evolution of (a) transverse beam halo and (b) transverse rms normalized emittance for $6 \sigma$ Gaussian, $3 \sigma$ Gaussian, parabolic, and 4D water bag input distributions, as a function of the beam current at the LEBT exit.

$$
\begin{gathered}
\epsilon_{i}=\sqrt{\left\langle r_{i}^{2}\right\rangle\left\langle r_{i}^{\prime 2}\right\rangle-\left\langle r_{i} r_{i}^{\prime}\right\rangle} \\
H=\frac{\sqrt{3\left\langle r_{i}^{4}\right\rangle\left\langle r_{i}^{\prime 4}\right\rangle+9\left\langle r_{i}^{2} r_{i}^{\prime 2}\right\rangle^{2}-12\left\langle r_{i} r_{i}^{\prime 3}\right\rangle\left\langle r_{i}^{3} r_{i}^{\prime}\right\rangle}}{2\left\langle r_{i}^{2}\right\rangle\left\langle r_{i}^{\prime 2}\right\rangle-\left\langle r_{i} r_{i}^{\prime}\right\rangle}
\end{gathered}
$$

where $r_{i}$ and $r_{i}^{\prime}(i=x, y, z)$ represents spatial and divergence coordinates of the particles in the beam respectively.

In the LEBT, the beam is at its lowest energy, and therefore the nonlinear space-charge force is at its highest. It is consequently essential to have a clear understanding of LEBT beam dynamics in the presence of nonlinear spacecharge fields and its interaction with the solenoid field, to minimize the emittance growth and beam halo formation. Here the degree of nonlinearity in the space-charge was varied using different input particle distributions. The emittance growth and halo development were observed for varying beam current for the chosen input distribution in the matched condition at the LEBT output.

Figure 3 shows the variation of beam emittance and halo parameter as a function of beam current, for four different initial beam distributions: 4D water bag, parabolic, Gaussian $6 \sigma$ and Gaussian $3 \sigma$ and identical input rms parameters. With an increase in beam current, the spacecharge force increases. Also, as we go from the 4D water bag to the Gaussian distribution (for a given current), the nonlinearity of the space-charge forces increases [Eq. (2)-(5)].

$$
\begin{gathered}
E(r)=\frac{I r}{2 \pi \epsilon_{0} R^{2} \beta c}[K V] \\
E(r)=\frac{2 I r}{3 \pi \epsilon_{0} R^{2} \beta c}\left(1-\frac{r^{2}}{3 R^{2}}\right)[4 \text { Dwaterbag }] \\
E(r)=\frac{3 I r}{4 \pi \epsilon_{0} R^{2} \beta c}\left(1-\frac{r^{2}}{2 R^{2}}+\frac{r^{4}}{12 R^{4}}\right)[\text { Parabolic }] \\
E(r)=\frac{I}{2 \pi \epsilon_{0} r \beta c}\left(1-e^{-2 \frac{r^{2}}{R^{2}}}\right)[\text { Gaussian }]
\end{gathered}
$$

Here $I$ is the beam current, $R$ is the beam size, $r$ is the radial distance from the center, and $\beta$ is the relative beam velocity.

The expressions for the electric fields for different beam distributions clearly show that the maximum nonlinearity is present in the Gaussian distribution, followed by the parabolic distribution. The 4D water bag distribution occupies the 6D hyper ellipsoid uniformly, and therefore the projection on the coordinate space shows a $-r^{2}+r^{4}$ dependence.

From Fig. 3 it is clear that the higher the nonlinearity, the greater the growth in emittance and the halo parameter. The emittance and halo parameters are most significant for the Gaussian, followed by parabolic and least for the 4D water bag distribution.

Our simulations show that the degree of nonlinearity has a substantial effect on beam emittance and halo. As space-charge nonlinearities set in, emittance blow-up commences. For the uniform and parabolic distributions, the increases in beam emittance and halo are still modest. For the Gaussian beam, however, there is a considerable increase in both quantities. It is, therefore, vital to understand the beam distribution coming out of the ion source. There is a tremendous advantage to having a beam that is close to the uniform distribution, and certainly not Gaussian. However, for our further simulations here, we have assumed a Gaussian $6 \sigma$ beam. This choice is based on experimental results at LEHIPA, where the actual beam distribution was measured to be close to Gaussian. A Gaussian beam distribution would also be the worst-case scenario that will provide an upper limit on the emittance growth and halo development along the linac.

As these matching channels are nonaccelerating structures, the vacuum criterion can be relaxed, and techniques like space-charge compensation [14] (SCC) can be used. The SCC technique requires the introduction of gas at slight pressure that gets ionized in the presence of the main beam. Generated electrons get accelerated toward the beam axis 


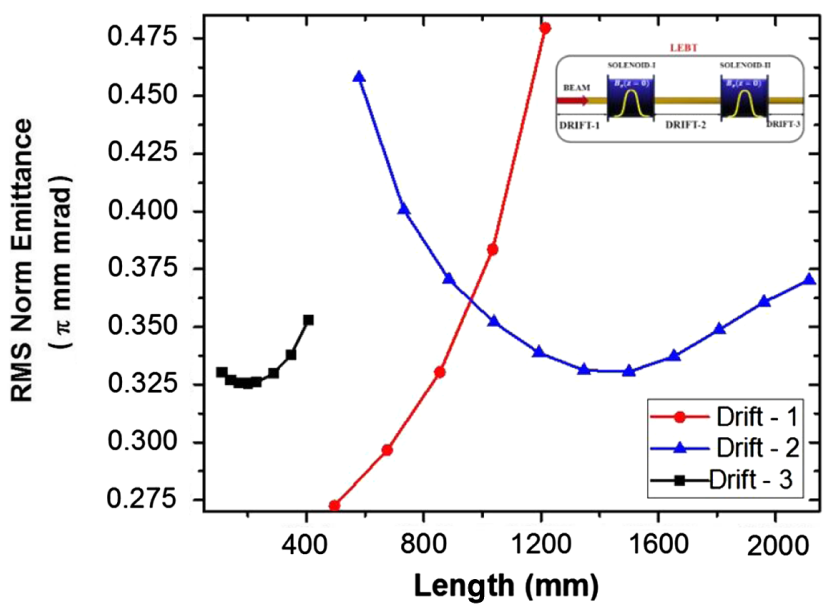

FIG. 4. RMS normalized beam emittance at the output of the LEBT for a matched beam as a function of drift-1, drift-2 and drift-3.

because of the potential well created by the proton beam. Therefore, they reduce the effective beam current and proportionally the space-charge force intensity in the transport line. Here we use a uniform space-charge compensation of $95 \%$ (corresponding to an effective beam current of $0.5 \mathrm{~mA}$ ) because, as demonstrated in [14] and [10], a space-charge compensation of $95 \%$ and higher has already been achieved. Further, our simulations show no beam quality variation beyond a space-charge compensation of $95 \%$.

The matching condition at the RFQ input dictates the strengths of the LEBT solenoids. Therefore, this constrains our ability to tune the LEBT solenoids to minimize the phase-space distortion of the beam. Besides solenoids, we also have varied the drift spaces before the solenoid (drift-1), in between the two solenoids (drift-2) and after the second solenoid (drift-3). For the worst-case scenario of a 6- $\sigma$ Gaussian distribution, the drift lengths have been optimized with the design beam current of $10 \mathrm{~mA}$ to keep minimum halo and emittance growth and also to be able to accommodate beam diagnostics, steerers, gate valves, bellows and vacuum pumps in the LEBT. The optimization curve showing the transverse beam emittance variation as a function of three drift lengths is shown in Fig. 4.

Fig. 4 suggests that there is an optimal drift length for drift-2 and drift-3, leading to a minimum value of beam emittance. As far as the drift-1 is concerned, longer the drift, the larger the beam at the solenoid input, and the beam requires tighter focusing to achieve the targeted Twiss parameters, leading to a higher emittance value. As drift-2 lies between the solenoids, the choice of its length determines the field strength of the second solenoid. A longer value of drift- 2 demands a higher magnetic field from the second solenoid and causes higher beam emittance. If we keep drift-2 too small, the beam size at the input to the second solenoid increases. Therefore, for given
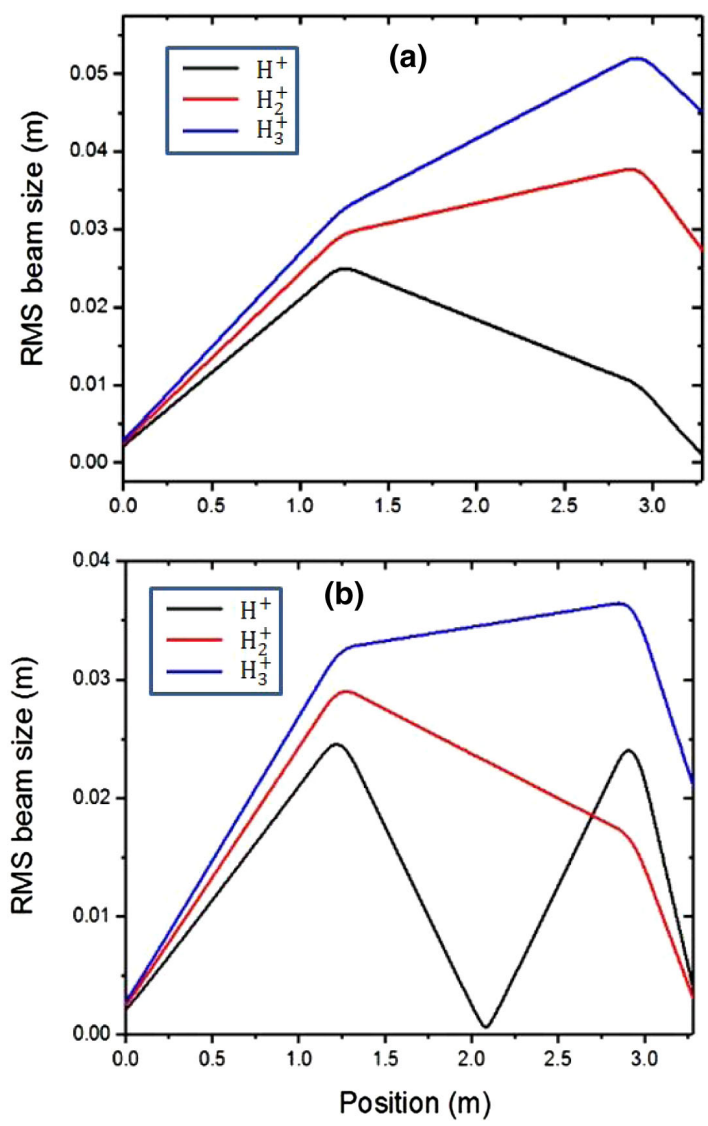

FIG. 5. Beam envelope for proton, $H_{2}^{+}$and $H_{3}^{+}$along the LEBT: (a) Scheme-I with gentle focusing and (b) Scheme-II with tighter focusing.

Twiss parameters at the RFQ input, solenoid-2 needs to provide stronger focusing due to which the beam suffers from space-charge issues in drift-3. The obtained optimal value for drift-2 is $1,400 \mathrm{~mm}$, giving an emittance of $0.326 \pi \mathrm{mm}$-mrad. Drift-3 is also optimized, and we obtain an optimal value of $200 \mathrm{~mm}$ for an emittance of $0.325 \pi \mathrm{mm}-\mathrm{mrad}$.

Besides the species of interest, that is protons, a fraction of other residual species like $\mathrm{H}_{2}^{+}$and $\mathrm{H}_{3}^{+}$are also present in the beam that is extracted from the ion source. Apart from matching and transport, LEBT can be used as a filter for these unwanted molecular ions by opting for an appropriate beam focusing scheme. The velocity-dependent focusing of the solenoid was used to separate the waist location for different species in the beam, and simulations were performed to study the behavior of the beam with multiple species, in two different focusing scenarios, as shown in Fig. 5(a) and Fig. 5(b).

In the first case, solenoid-1 performed weak focusing (magnetic field of $0.15 \mathrm{~T}$ ) and later the second solenoid (with a magnetic field of $0.26 \mathrm{~T}$ ). For the latter case, both solenoids were operated with a high magnetic field $(0.28 \mathrm{~T}$ and $0.34 \mathrm{~T}$ ); thus, the waist formation takes place between the two solenoids. One way to filter out the unwanted 


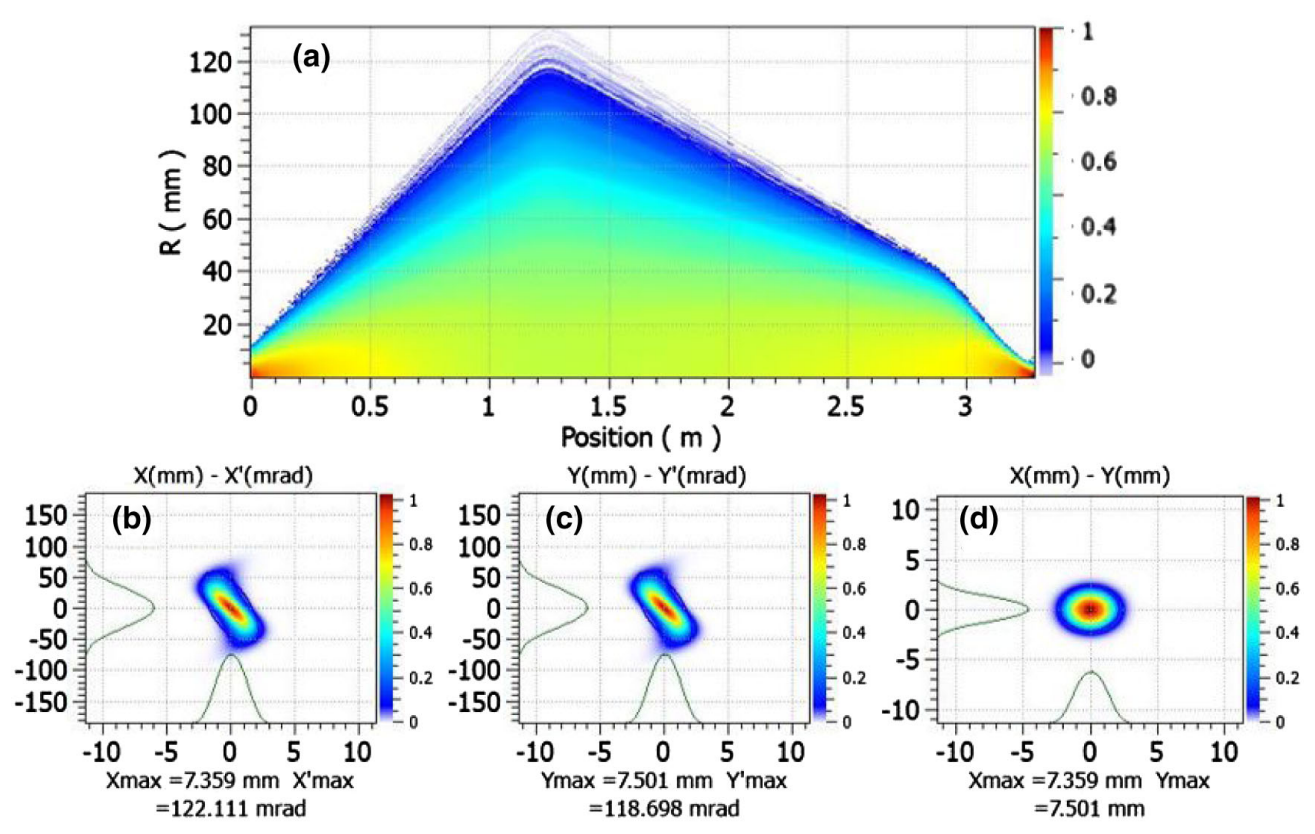

FIG. 6. (a) Particle density distribution in the $x-z$ plane along the MEHIPA LEBT, (b,c) phase space and (d) coordinate space particle density distribution in the transverse plane.

species is to put an aperture at the waist of the proton beam (beam radius $=5.7 \mathrm{~mm}$ ) where the beam size of $H_{2}^{+}$and $\mathrm{H}_{3}^{+}$is $23 \mathrm{~mm}$ and $35 \mathrm{~mm}$ respectively.

Our study suggests that the second scheme leads to transverse emittance growth from $0.2 \pi \mathrm{mm}$-mrad to $0.42 \pi \mathrm{mm}$-mrad ( $110 \%$ increase) because of strong beam focusing and therefore demands a compromise between the beam quality and beam purity. On the other hand, if we choose the first scheme, with weak focusing, the transverse emittance of the proton beam increases from $0.2 \pi \mathrm{mm}$-mrad to $0.23 \pi \mathrm{mm}$-mrad. The residual species will not be filtered out and will enter the RFQ, and will be filtered out there. If the proton fraction is high, the consequent beam loss should not be a problem. Therefore, considering the insignificant percentage of these species, and the lower emittance obtained, we have adopted the weak focusing scheme for the MEHIPA LEBT.

After the optimization of the LEBT geometry, solenoids fields, and the focusing scheme, the LEBT beam dynamics was performed with a $6 \sigma$ Gaussian distribution and a beam current of $10 \mathrm{~mA}$. As mentioned above, we have also employed space-charge compensation of $95 \%$ in the LEBT to reduce the space charge effects during the beam transport to the RFQ. The variation of the beam envelope and the particle distribution at the exit of the LEBT is shown in Fig. 6 and the final beam Twiss parameters and emittances are listed in Table-II.

As shown in Fig. 6(a), the beam size for the matched beam at the RFQ input reached its maximum at the entrance of the first solenoid with a beam size of $130 \mathrm{~mm}$, providing an estimate for the aperture. The beam experiences axisymmetric focusing and therefore maintains similar dynamics in both transverse planes with identical beam emittance and halo parameters. Figure 6(b) shows the phase-space and coordinate space particle density distribution at the exit of the LEBT. At the RFQ input, the beam obtains a transverse size of $7.35 \mathrm{~mm}$ and $7.50 \mathrm{~mm}$ in the $\mathrm{x}$ - and $\mathrm{y}$-directions respectively, with a maximum divergence of $122.25 \mathrm{mrad}$ and $118.6 \mathrm{mrad}$ in the $\mathrm{x}$ - and y-directions.

The next section discusses the acceleration of the matched beam through the RFQ.

\section{B. Radio frequency quadrupole (RFQ)}

The RFQ is known for its capability to focus, bunch, and accelerate the beam simultaneously and is most efficient at low energies $(\beta=0.01$ to 0.08$)$. The RFQ is also well

TABLE II. Final parameters of the MEHIPA LEBT and beam parameters at the exit.

\begin{tabular}{lc}
\hline \hline Parameter & Value \\
\hline Current $(\mathrm{mA})$ & 10 \\
Energy $(\mathrm{keV})$ & 50 \\
Drift 1 $(\mathrm{mm})$ & 1,000 \\
Drift 2 $(\mathrm{mm})$ & 1,400 \\
Drift 3 $(\mathrm{mm})$ & 180 \\
$B_{s 1}(\mathrm{~T})$ & 0.15 \\
$B_{s 2}(\mathrm{~T})$ & 0.26 \\
Solenoid lengths $(\mathrm{mm})$ & 300 \\
$\epsilon_{x, y}(\pi \mathrm{mm}-\mathrm{mrad})$ & 0.23 \\
$\alpha_{x, y}$ & 0.80 \\
$\beta_{x, y}(\mathrm{mrad} / \pi \mathrm{mm})$ & 0.04 \\
\hline \hline
\end{tabular}



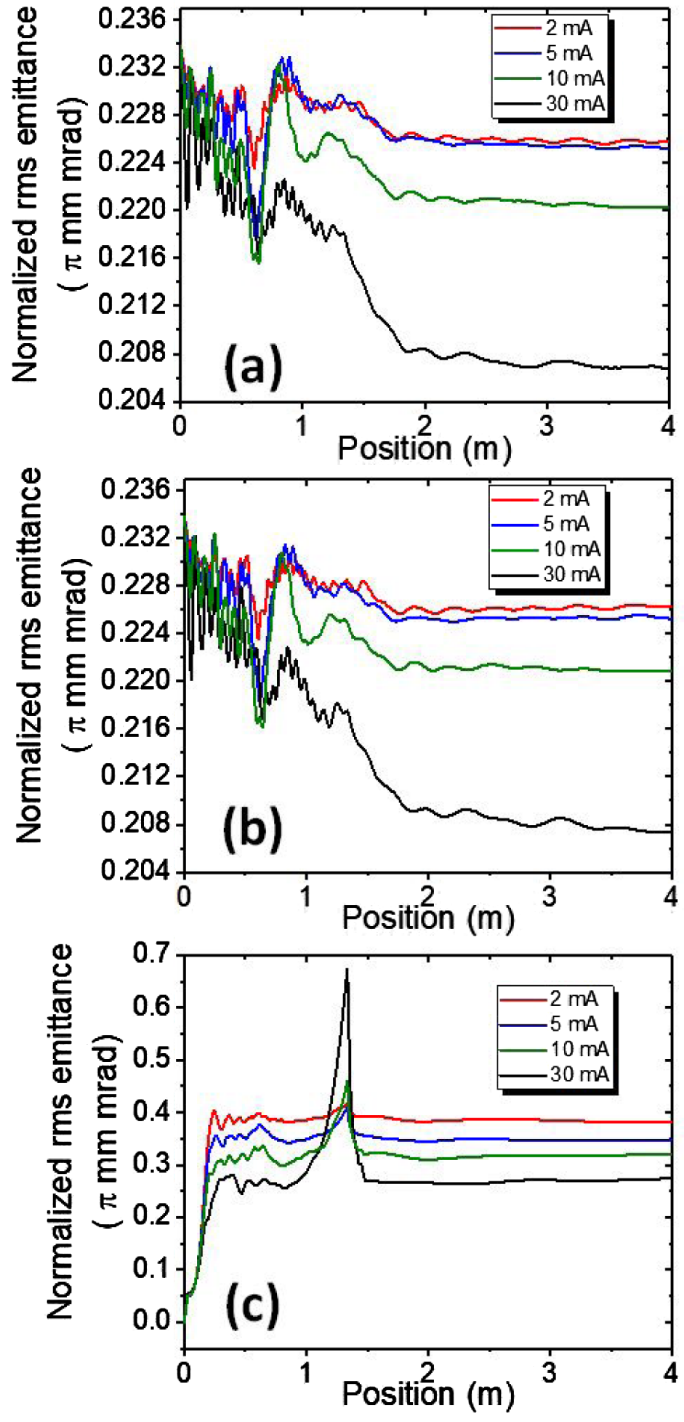

FIG. 7. Variation of the (a) xx', (b) yy' and (c) zz' normalized RMS emittance along the RFQ for different beam currents.

known for its efficiency in terms of high transmission and low emittance growth. Considering these advantages a $4 \mathrm{~m}$ long, $325 \mathrm{MHz}$ RFQ with a constant vane voltage of $80 \mathrm{kV}$ and with the peak surface field less than 1.8 times the Kilpatrick limit was designed with conventional design protocols [15], to accelerate a $50 \mathrm{keV}, 10 \mathrm{~mA}$, matched proton beam to $3 \mathrm{MeV}$. Here our RFQ design criterion was to maximize the beam transmission and minimize the emittance growth.

3D particle-in-cell (PIC) [16] beam dynamics simulations were performed using the computer code TOUTATIS [17] to study different design philosophies for the RFQ. As discussed earlier, beam current plays a significant role in determining the beam quality. Therefore, we investigated the beam dynamics along with the RFQ for the beam current of $2 \mathrm{~mA}, 5 \mathrm{~mA}, 10 \mathrm{~mA}$, and $30 \mathrm{~mA}$ but with identical input rms parameters. The variation of beam

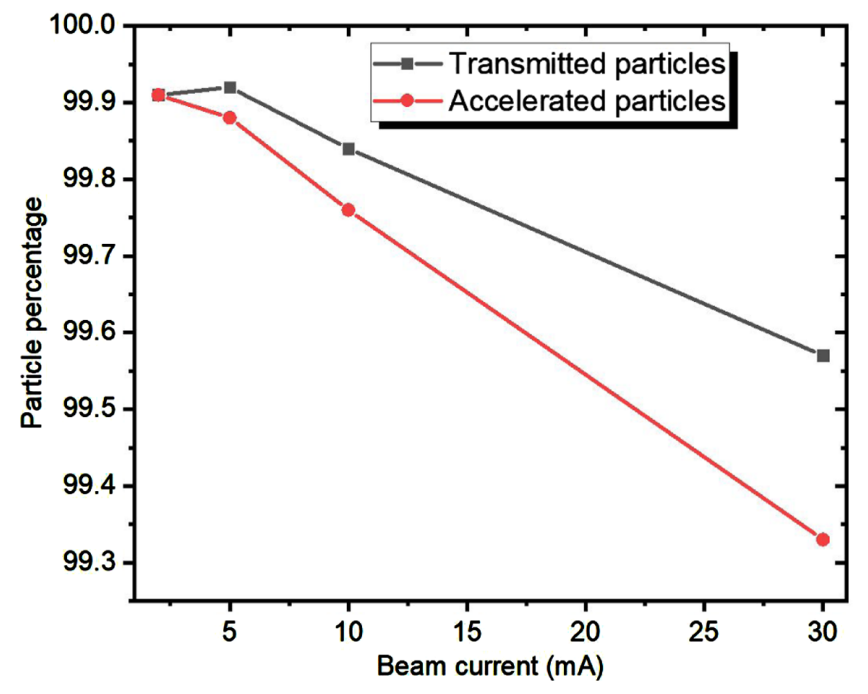

FIG. 8. Beam transmission (transmitted and accelerated partices) through the RFQ as a function of beam current.

emittance along the RFQ for different currents is shown in Figs. 7 and the dependence of beam transmission on beam current is shown in Fig. 8 .

As we increase the beam current from $2 \mathrm{~mA}$ to $30 \mathrm{~mA}$, the percentage of transmitted as well as accelerated particles decreases from $99.91 \%$ to $99.57 \%$ and from $99.91 \%$ to $99.33 \%$ respectively. The transverse emittance shows a decrease from $0.226 \pi \mathrm{mm}$-mrad to $0.207 \pi \mathrm{mm}$ mrad in both, the $\mathrm{x}$ - and $\mathrm{y}$-direction, because of particle loss. The longitudinal emittance shows a monotonic decrease with increasing beam current with a maximum value of $0.383 \pi \mathrm{mm}-\mathrm{mrad}$ for a beam current of $2 \mathrm{~mA}$, and a minimum of $0.266 \pi \mathrm{mm}$-mrad for a beam current of $30 \mathrm{~mA}$. Fig. 8 also suggests that for the design beam current of $10 \mathrm{~mA}$, the percentage of accelerated particles is $99.76 \%$, and even with a three-fold increase in the beam

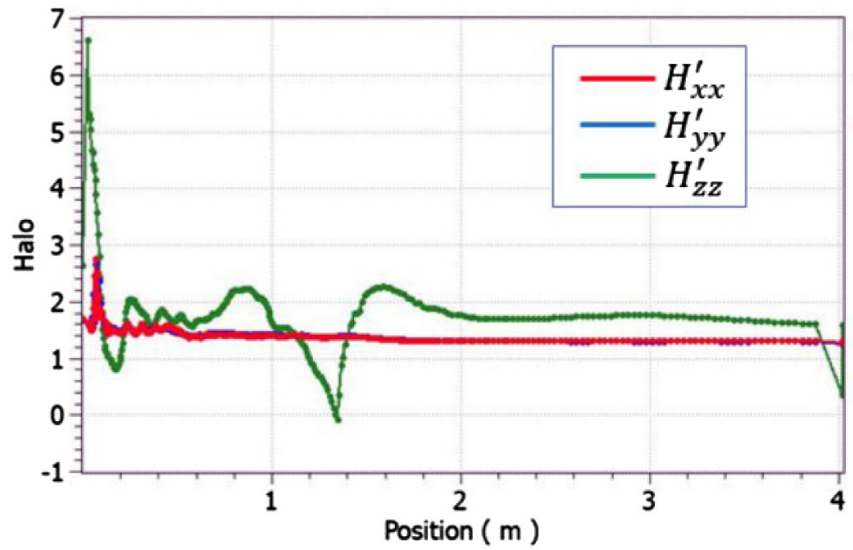

FIG. 9. Variation of transverse and longitudinal beam halo parameter along with the RFQ for the designed beam current of $10 \mathrm{~mA}$. 
TABLE III. Final RFQ parameters.

\begin{tabular}{lc}
\hline \hline Parameter & Value \\
\hline Input energy $(\mathrm{MeV})$ & 0.05 \\
Output energy $(\mathrm{MeV})$ & 3.0 \\
Duty factor & $100 \%$ \\
Frequency $(\mathrm{MHz})$ & 325 \\
Beam current $(\mathrm{mA})$ & 10 \\
rf power $(\mathrm{kW})$ & 400 \\
Vane voltage $(\mathrm{kV})$ & 80 \\
Synchronous phase & -30 \\
Transmission & $99 \%$ \\
Output $\epsilon_{x}(\pi$ mm-mrad$)$ & 0.220 \\
Output $\epsilon_{y}(\pi \mathrm{mm}-\mathrm{mrad})$ & 0.220 \\
Output $\epsilon_{z}(\pi \mathrm{mm}-\mathrm{mrad})$ & 0.312 \\
Length $(\mathrm{m})$ & 4 \\
\hline \hline
\end{tabular}

current, the beam transmission reduces by only $0.43 \%$, demonstrating an efficient RFQ design.

We also looked at the variation of the beam halo parameter [Eq. (3)] for the design beam current of $10 \mathrm{~mA}$ and the result is shown in Fig. 9. With a transverse matched beam at the input, the transverse halo parameter grows from 1 to 1.4. As far as the longitudinal halo parameter is concerned, it shows an erratic behavior because of beam bunching in RFQ with a final value of 0.3 at the RFQ exit.

The final parameters for the designed RFQ are listed in Table III. The evolution of the beam envelope through the RFQ and the output particle density distributions in phasespace and coordinate space are shown in Figs. 10(a) and 10(b) respectively, for a $95 \%$ neutralized beam in the LEBT. At a beam current of $10 \mathrm{~mA}$, we obtain a transverse beam size of $2.6 \mathrm{~mm}$ and a longitudinal length of $4 \mathrm{~mm}$. The transverse divergences at the exit of the RFQ have been minimized to avoid the beam blow-up in the medium
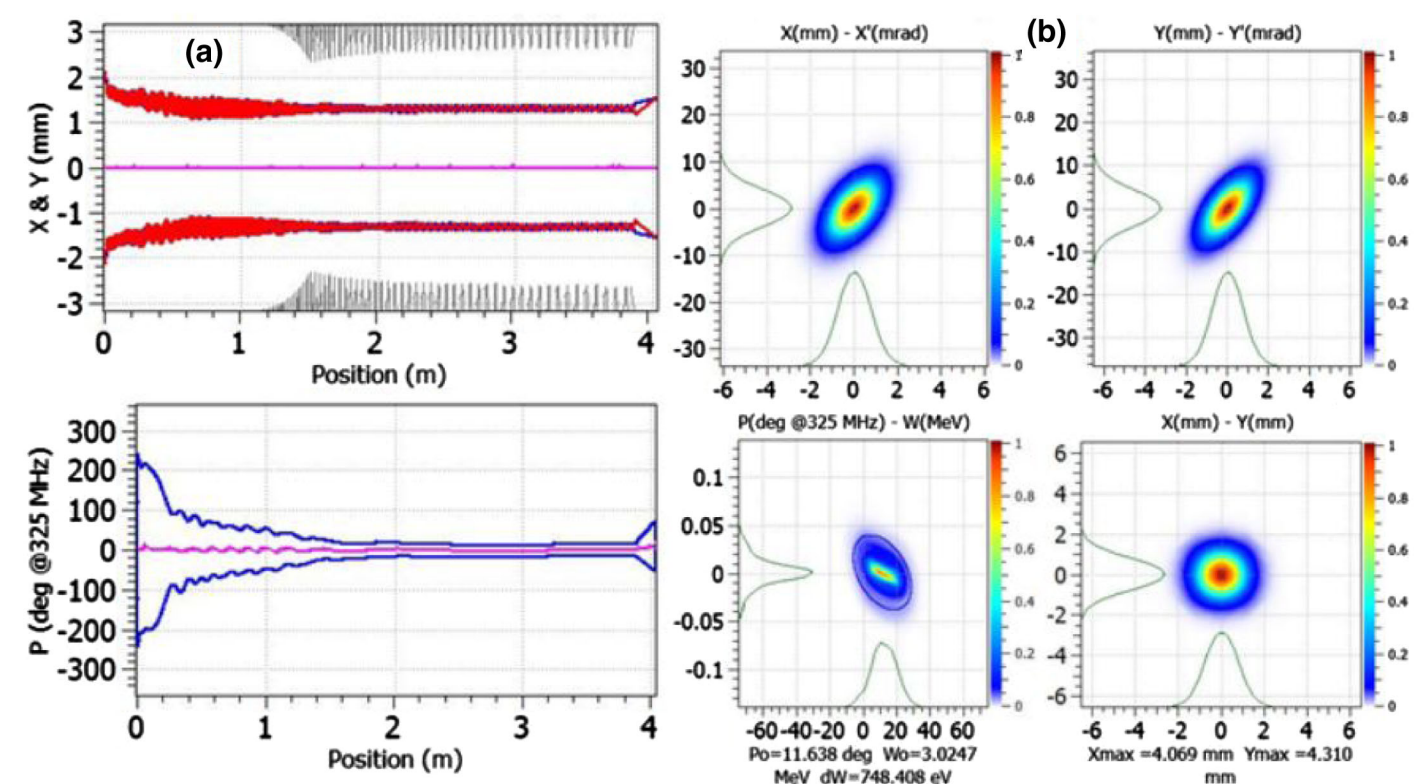

FIG. 10. (a) Beam envelope ( $x$ in red and $y$ in blue) along the RFQ, and variation of beam phase width along RFQ, and (b) phase space (x-x', y-y' and phase-energy) and coordinate (x-y) space particle distribution at the exit of RFQ, for the beam current of $10 \mathrm{~mA}$.

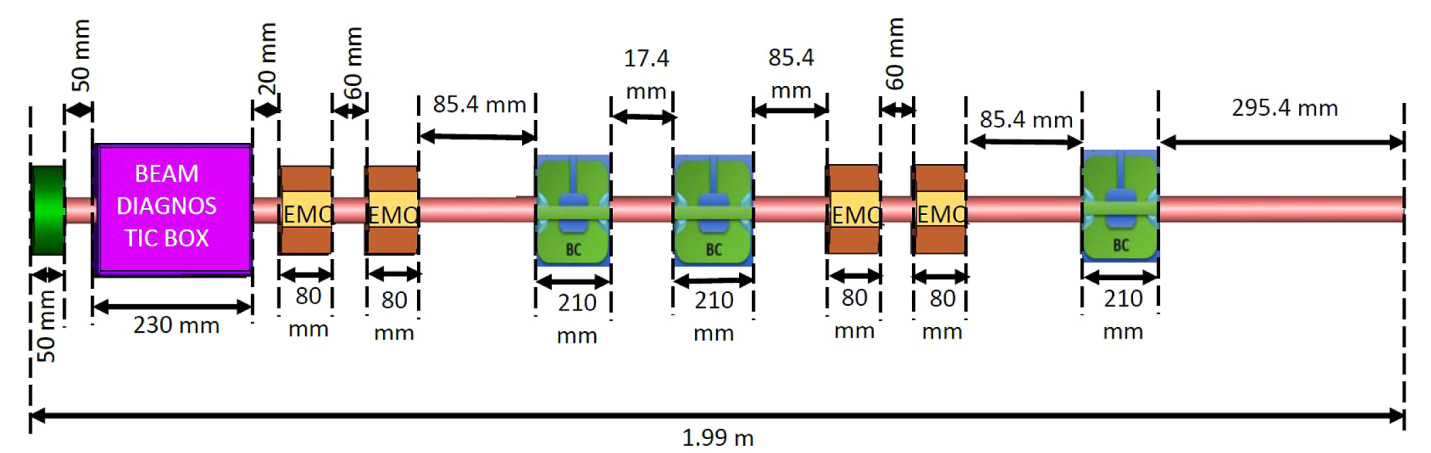

FIG. 11. Layout of the MEBT demonstrating the position and dimensions of the quadrupole doublets and buncher cavities. 
TABLE IV. MEBT parameters.

\begin{tabular}{lc}
\hline \hline Parameter & Value \\
\hline Total length $(\mathrm{m})$ & 1.99 \\
Quadrupole 1 gradient $(\mathrm{T} / \mathrm{m})$ & -17.20 \\
Quadrupole 2 gradient $(\mathrm{T} / \mathrm{m})$ & 17.27 \\
Quadrupole 3 gradient $(\mathrm{T} / \mathrm{m})$ & -15.52 \\
Quadrupole 4 gradient $(\mathrm{T} / \mathrm{m})$ & 12.89 \\
Gap 1 $E_{0} \mathrm{TL}(\mathrm{kV})$ & 15.03 \\
Gap 2 $E_{0} \mathrm{TL}(\mathrm{kV})$ & 49.17 \\
Gap 3 $E_{0} \mathrm{TL}(\mathrm{kV})$ & 12.81 \\
Output $\epsilon_{x}(\pi \mathrm{mm}-\mathrm{mrad})$ & 0.228 \\
Output $\epsilon_{y}(\pi \mathrm{mm}-\mathrm{mrad})$ & 0.224 \\
Output $\epsilon_{z}(\pi \mathrm{mm}-\mathrm{mrad})$ & 0.317 \\
\hline \hline
\end{tabular}

energy beam transport channel and have a final value of $10 \mathrm{mrad}(\mathrm{rms})$ in both $\mathrm{x}$ - and $\mathrm{y}$-directions.

\section{MEDIUM ENERGY BEAM TRANSPORT (MEBT)}

The $3 \mathrm{MeV}$ beam from the RFQ is transported to the superconducting section of MEHIPA using a medium energy beam transport (MEBT) channel. The MEBT channel uses two electromagnetic quadrupole doublets to tune the transverse Twiss parameters of the beam and three buncher cavities to perform matching in the longitudinal direction. The proposed MEHIPA MEBT will be implemented with a diagnostic box at its input. Here we plan to use a buncher cavity with varying voltages for the measurement of longitudinal emittance. The emittance measurement in the transverse plane will be carried out using a wire scanner. Each quadrupole set is accompanied by corrector magnets to keep the beam on-axis. Finally, toward the end of the MEBT channel, we plan to employ a setup to deploy desired diagnostic elements like Faraday cup, wire scanner, etc. The schematic of the proposed MEBT is shown in Fig. 11.

As shown in Fig. 11, a third buncher cavity was used after the first quadrupole doublet, whose location and field can be tuned to optimize the transverse aspect ratio of the beam and improve longitudinal beam quality. The superconducting section of the linac uses superconducting solenoids to confine the beam in the transverse plane and demands an axisymmetric beam from the MEBT, and therefore the additional buncher cavity plays a significant role in the MEBT design.

The design of the buncher cavity for the recommended MEBT considers two rf gaps. As the voltage requirement from such cavities reaches a value of $100 \mathrm{kV}$, a two-gap buncher is preferred over a single gap buncher to reduce the voltage requirement from a single gap. As far as transverse focusing is concerned, a quadrupole doublet is preferred over the triplet configuration to minimize the length of the MEBT. The fields for the two quadrupole doublets and the buncher cavities (2 and 3) were varied independently to achieve the acceptance beam ellipse at the input of the SC section of the linac. With the provision made to accommodate beam diagnostic and steering elements, the total MEBT length reaches a value of $1.99 \mathrm{~m}$.

A careful optimization was performed to match the beam from the RFQ to the input of the SC section of the linac. The Twiss parameters were tuned while obtaining an axisymmetric beam and minimizing the emittance growth and halo blow-up. The final quadrupole gradients and buncher fields are listed in Table IV.

Figure 12 and Fig. 13 show the results of beam dynamics simulations performed with the optimized MEBT fields and (a)
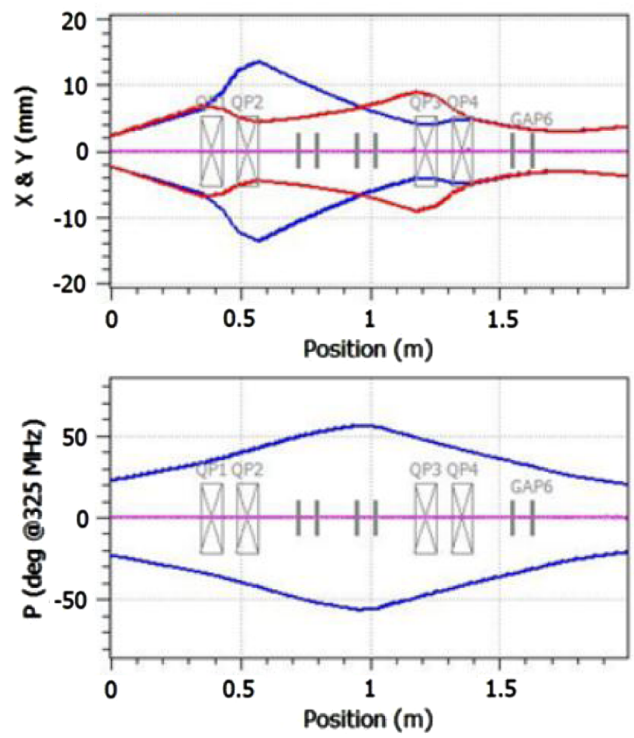

$X(\mathrm{~mm}) \cdot \mathrm{X}^{\prime}(\mathrm{mrad})$
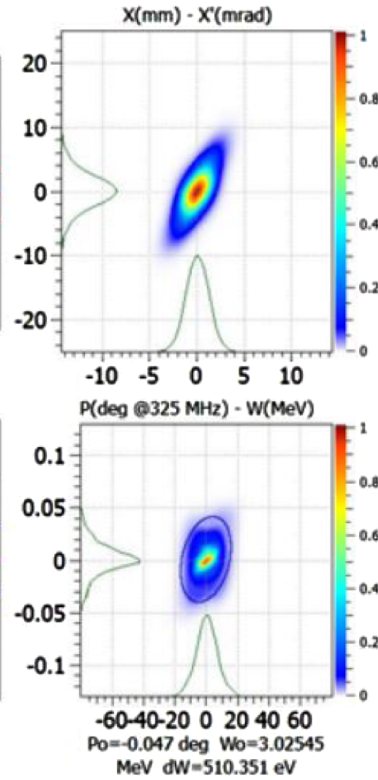

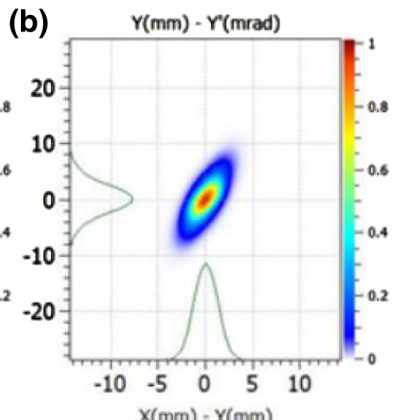

$X(\mathrm{~mm})=Y(\mathrm{~mm})$

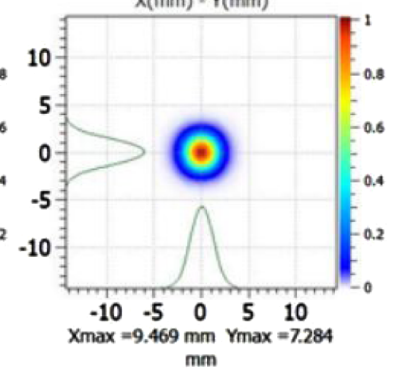

FIG. 12. (a) x, y, and phase envelope along the MEBT, (b) x-x', y-y', and phase-energy distribution at the exit of the MEBT. 

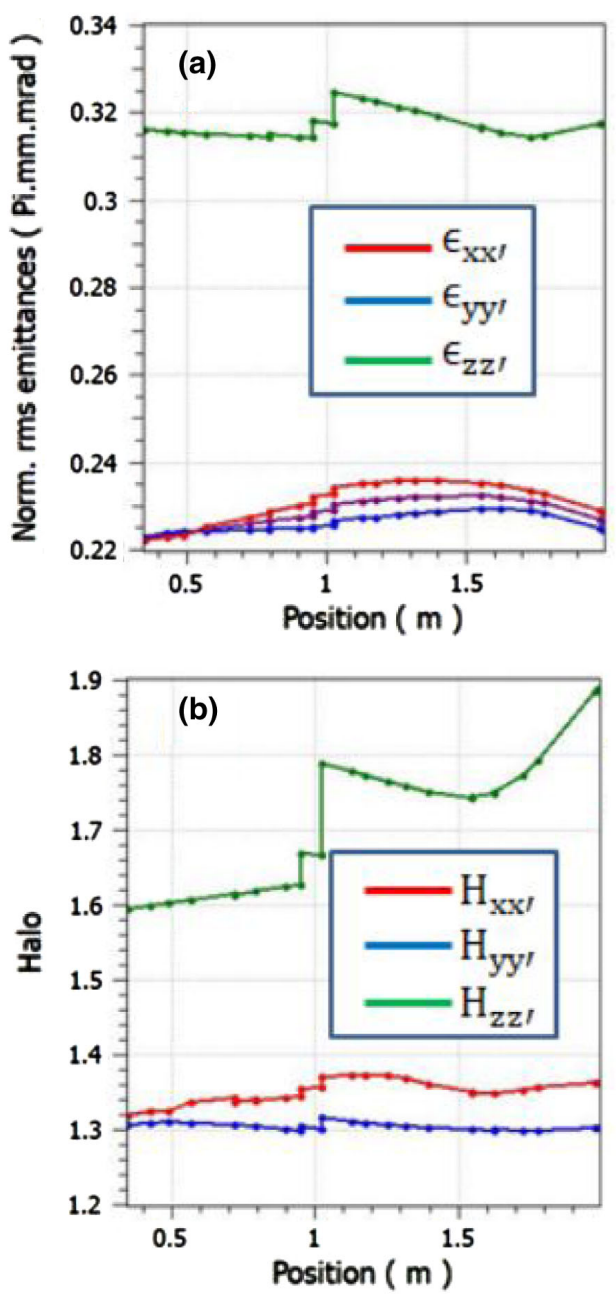

FIG. 13. (a) RMS normalized transverse and longitudinal emittance along MEBT, and (b) Transverse and longitudinal beam halo parameter along MEBT.

geometric parameters. It can be seen from Fig. 12(a) that we obtain a circular beam in the transverse plane at the exit of the MEBT with an rms size of $1.76 \mathrm{~mm}$ in both $\mathrm{x}$ - and y-directions. Figure 12 also shows the variation of the longitudinal size of the beam. The transverse beam sizes provide an estimate of the aperture, but the emittance and the halo parameter determine the beam quality. The evolution of these two parameters is shown in Figs. 13(a) and (b) respectively. We observe an emittance growth of $3.63 \%, 108 \%$, and $-1.6 \%$ in the $\mathrm{x}-, \mathrm{y}-$, and z-directions, respectively. Since the halo is defined as a ratio of the fourth moment to the second moment, it is sensitive to the tails of the distribution. Figure 13(b) shows the variation in the halo parameter of the beam along the MEBT. Here we observe a halo growth from 1.317 to 1.362 , from 1.305 to 1.302 , and from 1.594 to 1.887 , corresponding to a percentage growth of $3.4 \%,-0.22 \%$, and $18.3 \%$, in the xx', yy', and $\mathrm{zz}^{\prime}$ planes respectively. The blow-up in the longitudinal halo is confirmed from the phase space distribution shown in Fig. 12(b) where one can observe the initiation of the filamentation in the zz' phase-space leading to a higher weight to the fourth moment of the beam and therefore increasing the longitudinal beam halo. The transverse phase-space does not show any distortion in the particle distribution leading to an insignificant halo growth.

The first rf gap is placed at $700 \mathrm{~mm}$ from the RFQ. This length is necessary to provide space for beam diagnostics and the first transverse focusing doublet. Provision for transverse beam focusing has been made first because a transverse beam blow-up would immediately manifest in terms of beam loss. This long region devoid of longitudinal focusing has an adverse effect on longitudinal beam phase space.

The optimized MEBT beam dynamics provide us an acceptable beam quality with an insignificant distortion from the point of view of transverse and longitudinal emittance and halo parameters. The requirement of the quadrupole gradient and the buncher cavity fields are well below the limitation posed by the heating of the copper coils and the breakdown due to the voltage in the rf gap. The next section discusses the transport of the matched beam through the SC section of MEHIPA.

\section{SUPERCONDUCTING INJECTOR SECTION}

Single spoke resonators (SSRs) [18-26] are known to be efficient at lower velocity range and are increasingly being used worldwide in high-intensity hadron accelerators [27-30] and therefore are chosen for beam acceleration from $3 \mathrm{MeV}$ to $200 \mathrm{MeV}$. Here we have chosen three families of spoke cavities, namely SSR-A, SSR-B, and SSR-C operating at $325 \mathrm{MHz}$. The design for these single spoke cavities had been performed in two stages; the first stage comprises the minimization of the number of these cavities [31] (while keeping the length of the cavity fixed) for a given energy range by determining an optimal $\beta$ for these cavities. The second stage constitutes the rf design and optimization of these cavities to satisfy the functional requirement of the linac [6]. Besides, we have also looked at issues like multipacting [32] and the effects of higher-order modes [32] in the spoke cavity in detail and quantified them in terms of multipacting growth rate and $\mathrm{R} / \mathrm{Q}$, respectively. Here we have used the code GenLinWin [33] for the lattice design and optimization of the longitudinal parameters. As far as transverse confinement of the beam is concerned, MEHIPA uses superconducting solenoids [34,35]. These solenoids are designed and optimized using the magneto-static solver of CST Studio Suite [36]. Three-dimensional solenoid and cavity fields were used to perform fully three-dimensional PIC beam dynamics simulations for the SC section of MEHIPA linac. Finally, we have studied the linac tolerances with the introduction of beam, cavity, and magnet errors.

\section{A. $\beta_{\text {opt }}$ optimization for SSR-A, SSR-B, and SSR-C}

One of the chief concerns of a linac design is its length, which is determined by the number of accelerating cavities 


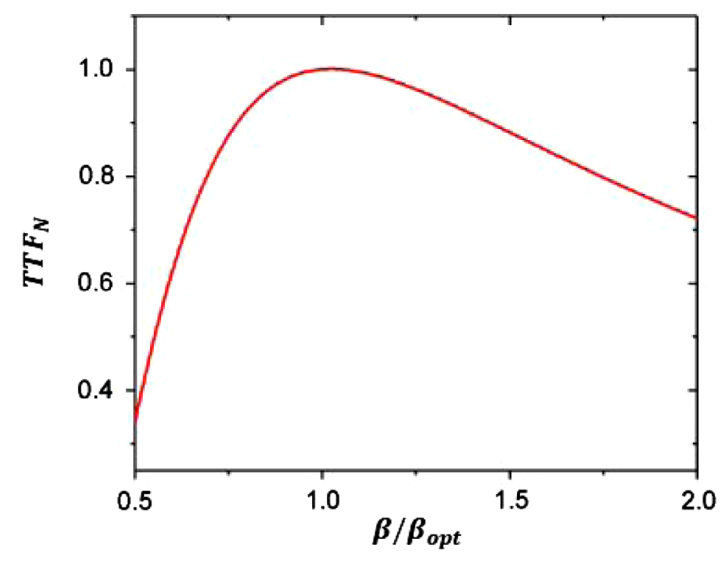

FIG. 14. Universal normalized transit time factor as a function of normalized $\beta$.

used for a given energy range. A possible way to minimize the number of these cavities and, therefore, the overall length of the linac is to optimize the cavity $\beta$ for which the maximum acceleration is achieved. The value of this optimized $\beta$ is always higher than the geometric $\beta$ [37] (gap to gap distance $=\beta_{g} \lambda / 2$ ) of the cavity and is called optimal $\beta\left(\beta_{\text {opt }}\right)$. The $\beta$ optimization process requires the universal transit time factor for the spoke cavities and therefore, to begin with, we designed our cavities with a value of $\beta_{g}$ that is in the middle of the energy range for the given family of SSR, and we chose the values as $0.11,0.21$ and 0.46 for SSR-A, SSR-B, and SSR-C respectively. The axial fields from these cavities were used to obtain the transit time factors for each family of spoke cavities and were later normalized with respect to the maximum transit time factor (y-axis) and $\beta_{\text {opt }}$ (x-axis) to obtain a universal function that is the same for all three families of spoke resonators [38]. The functional form of the normalized transit time factor, $T_{N}(\beta)$, was obtained by fitting this universal curve and can be written as,

$$
T_{N}(\beta)=\frac{-1.52+3.724 \frac{\beta}{\beta_{\mathrm{opt}}}}{1-\frac{1.203 \beta}{\beta_{\mathrm{opt}}}+2.4147 \frac{\beta}{\beta_{\mathrm{opt}}}} .
$$

Figure 14 plots this polynomial expression as a function of normalized value, $\beta / \beta_{\mathrm{opt}}$.

Using this figure one can calculate the number of cavities needed for the energy gain from $3 \mathrm{MeV}$ to $10 \mathrm{MeV}$, $10 \mathrm{MeV}$ to $50 \mathrm{MeV}$ and $50 \mathrm{MeV}$ to $200 \mathrm{MeV}$ (SSR-A, SSR-B, and SSR-C respectively) by changing the optimal beta of the cavities. The variation in the cavity number as a function of $\beta_{\text {opt }}$ is shown in Fig. 15.

As shown in Fig. 15, the number of cavities is minimum for a given range of optimal beta for SSR-A, followed by for SSR-B and finally for the SSR-C section of the linac. For SSR-A, the optimal beta range for which the number of cavities is minimum ranges from 0.123 to 0.155 ; for SSR-B,
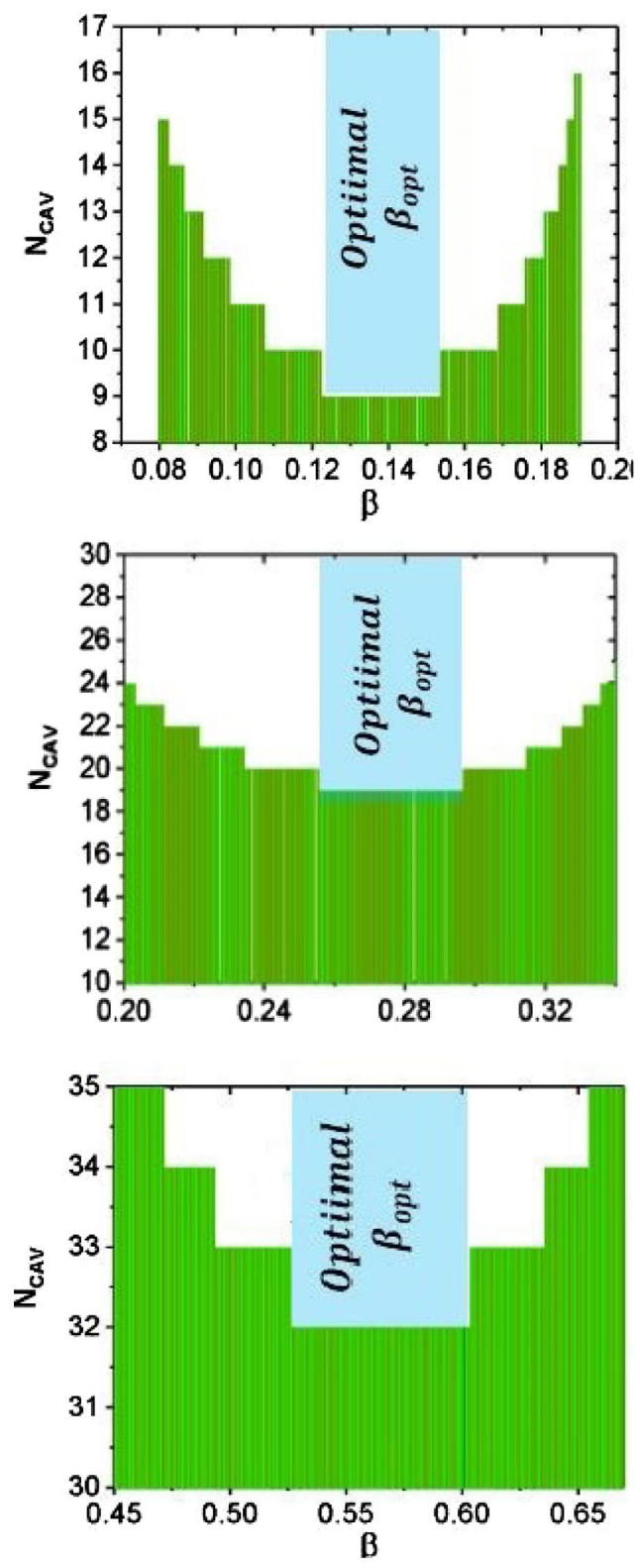

FIG. 15. Number of accelerating cavities and corresponding linac length as a function of $\beta_{\text {opt }}$ for SSR-A, SSR-B, and SSR-C.

it is between 0.245 and 0.292 , and for SSR-C, it is between 0.534 and 0.603 , with 9,19 and 32 cavities respectively.

$\mathrm{RF}$ design simulations were performed by varying the cavity geometric parameters to obtain the desired $\beta_{\text {opt }}$, simultaneously satisfying the functional requirement. For a given geometry, $\beta_{\text {opt }}$ of the cavity can be varied by changing the spoke equator thickness that, in turn, changes the iris length. For all these simulations, we keep the cavity length fixed, and the spoke equator dimensions were used to change $\beta_{\text {opt }}$ of the cavity.

\section{B. rf design and optimization}

The electromagnetic simulations for the SSRs were performed using CST Microwave Studio [36] with over 


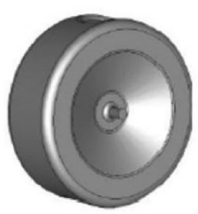

SSR-A

3-10 MeV

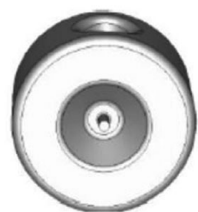

SSR-B

10-50 MeV

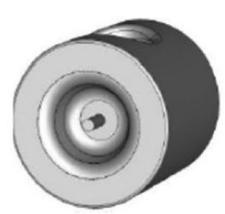

SSR-C

50-200 MeV
FIG. 16. Vacuum model of SSR-A, SSR-B, and SSR-C cavities.

0.8 million mesh cells, and the cavities were tuned to operate at $325 \mathrm{MHz}$. Figure 16 shows the vacuum model of the cavities. Though the cavity frequency was tuned, the peak surface electric field near the iris [Fig. 17(a)] and the peak surface magnetic field near the spoke base (Fig. 17(b)) are observed to exceed the functional requirement of $40 \mathrm{MV} / \mathrm{m}$ and $70 \mathrm{mT}$ respectively.

One of the primary objectives of the rf design is to minimize the peak surface electric field to avoid field emission and also to minimize the peak surface magnetic field to prevent the loss of superconductivity. Figure 18 shows the nomenclature of the different geometric parameters that we have chosen for the optimization of the spoke resonator geometry.
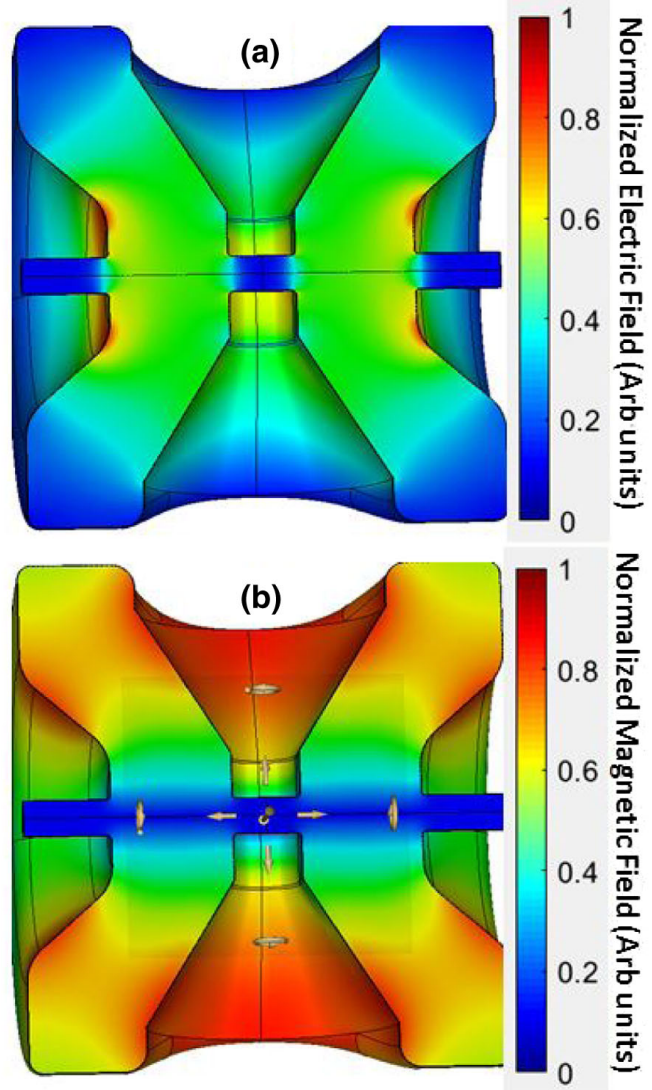

FIG. 17. (a) Electric and (b) magnetic field distribution in SSR-C cavity.
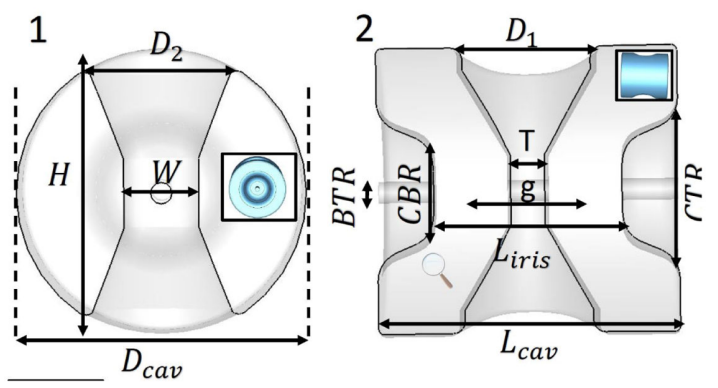

FIG. 18. Nomenclature used for the various geometric parameters of the spoke cavities.

The minimization studies were preceded by systematic sets of simulations to measure the dependence of the parameters like peak surface electric field, peak surface magnetic field and shunt impedance on various geometric parameters like spoke base major radius $\left(D_{1}\right)$, spoke base minor radius $\left(D_{2}\right)$, spoke equator major radius (W), spoke equator minor radius $(\mathrm{T})$, and the spoke height $(\mathrm{H})$. The study shows that the electric field is maximum on-axis, and therefore the peak electric field is affected by axial geometric parameters like $\mathrm{T}$ and $\mathrm{W}$. The magnetic field is maximum near the spoke base and therefore spoke base geometry affects the peak magnetic field. The spoke height $(\mathrm{H})$ could be used to tune the frequency of the cavity.

Spoke base parameters $D_{1}$ and $D_{2}$ were varied to minimize the peak surface magnetic field and spoke equator parameters $T$ and $W$ were varied to minimize the peak surface electric field. The detuning occurring due to variation in the spoke base and equator geometric parameters were corrected using the spoke height $(H)$. Besides the minimization of the peak surface fields, the cavity's R/Q was kept higher for better acceleration efficiency. As the electromagnetic design involves several rf parameters, global optimization was performed to obtain a balanced rf design.

After several iterations, the minimum values of $E_{\text {peak }} /$ $E_{\text {acc }}$ and $B_{\text {peak }} / E_{\text {acc }}$ were achieved for each family of spoke resonators and are listed in Table V. For the minimum peak field design, the R/Q for SSR-A, SSR-B, and SSR-C were found to be $129 \Omega, 223 \Omega$, and $322 \Omega$ respectively. The axial electric field from the optimized rf cavity design was used to calculate the transit time factor for all three SSR families and is shown in Fig. 19.

As Fig. 15 suggests, the optimal $\beta$ for these cavities is found to be in a range of 0.123 to $0.155,0.245$ to 0.292 , and 0.534 to 0.6 for SSR-A, SSR-B, and SSR-C respectively. Our study showed that the value of spoke equator geometry for which we achieve the lowest possible $\beta_{\text {opt }}$ leads to higher peak surface electric field. The variation of the peak electric field ratio for change in the optimal beta is shown in Fig. 20. Figure 20 suggests that with the decrease in the optimal beta value, the peak electric field ratio increases and crosses the limit of $40 \mathrm{MV} / \mathrm{m}$ and therefore we settle 
TABLE V. Final rf and geometric parameters for the SSR-A, SSR-B, and SSR-C cavities.

\begin{tabular}{lccc}
\hline \hline Parameters & SSR-A & SSR-B & SSR-C \\
\hline Aperture radius $(\mathrm{cm})$ & 1.5 & 1.5 & 2.0 \\
Effective Length $(\mathrm{cm})$ & 13.29 & 23.63 & 49.66 \\
$\mathrm{~T}(\mathrm{~cm})$ & 2.3 & 4.41 & 3.81 \\
$\mathrm{~W}(\mathrm{~cm})$ & 4.06 & 7.2 & 6.29 \\
$D_{1}(\mathrm{~cm})$ & 10.2 & 7.6 & 13.1 \\
$D_{2}(\mathrm{~cm})$ & 11.2 & 5 & 13.15 \\
$R / Q(\Omega)$ & 129 & 223 & 322 \\
$E_{\text {peak }} / E_{\text {acc }}$ & 4.58 & 3.86 & 3.44 \\
$B_{\text {peak }} / E_{\text {acc }}(\mathrm{mT} /(\mathrm{MV} / \mathrm{m}))$ & 8.69 & 6.87 & 5.74 \\
Accelerating gradient & 8 & 10 & 11 \\
$\mathrm{G}(\Omega)(\mathrm{MV} / \mathrm{m})$ & 48 & 139 & 128 \\
\hline \hline
\end{tabular}

with a $\beta_{\text {opt }}$ value of $0.144,0.257$ and 0.543 for SSR-A, SSR-B, and SSR-C respectively, for which the surface fields are within the functional requirements of the cavity.

\section{HOM analysis}

The eigensolutions for a cavity form a complete orthogonal set, and we can treat the individual excitations separately. Excitation of the cavity higher-order modes (HOMs) causes excess heat dissipation on the cavity walls and puts an undesirable load on the cryogenics and therefore requires HOM dampeners. Especially for high-intensity beams, if the frequency component of the bunch spectrum falls in resonance with these HOMs, the beam becomes unstable, and beam transmission, as well as the superconductivity of the cavity, are compromised. Studies performed by S. Kim, et al. [39] clearly suggest that a resonant power build-up because of these HOMs may lead to excess heat generation in the superconducting cavities. Here we have studied the dominant monopole, dipole and quadrupole modes for SSR-A, SSR-B, and SSR-C, in terms of their $R / Q$. The $R / Q$ of the monopole mode as a function of $\beta$ is plotted in Fig. 21 for SSR-A, SSR-B, and SSR-C,

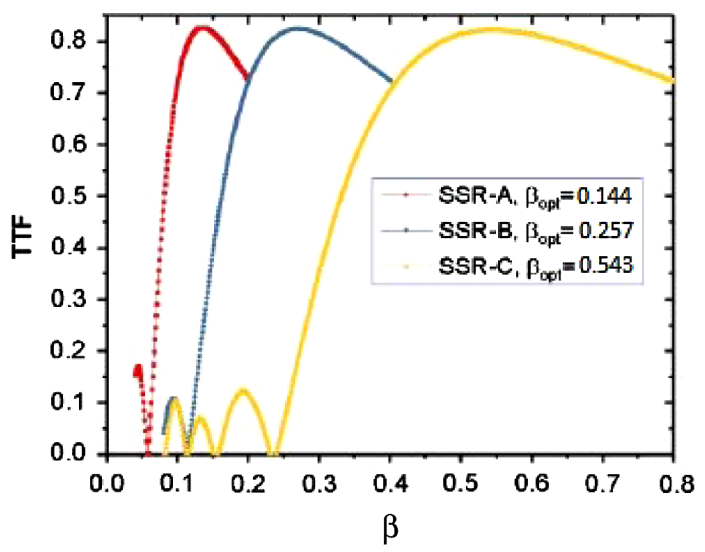

FIG. 19. Transit time factor as a function of $\beta$ for the SSR-A, SSR-B, and SSR-C cavities.
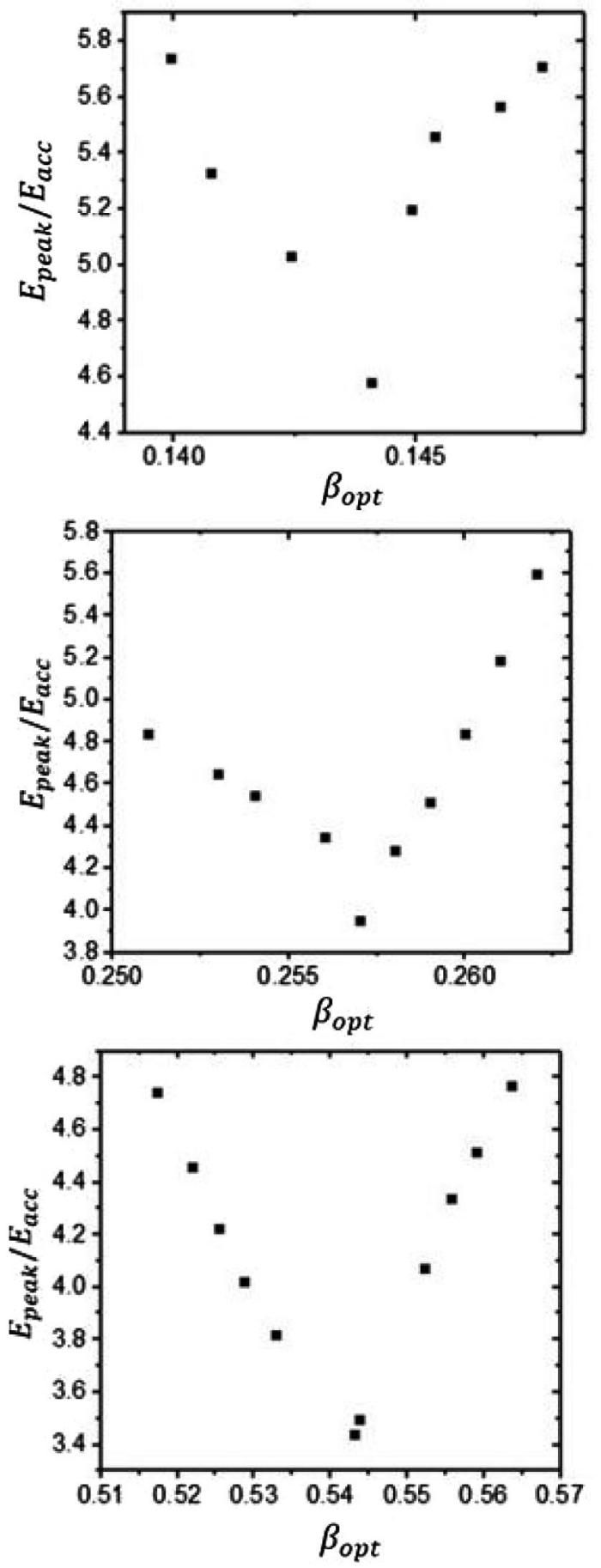

FIG. 20. Variation of peak field ratio with the change in the optimal beta of the cavity.

respectively. It can be seen that in each case at low $\beta$, the TM mode with frequency $422.03 \mathrm{MHz}, 397.96 \mathrm{MHz}$ and 339.33 MHz, for SSR-A, SSR-B, and SSR-C, respectively, dominates over the operating frequency of $325 \mathrm{MHz}$. However, the R/Q for these modes quickly damps down with increasing $\beta$. Also, these frequencies are 97, 73 and $14 \mathrm{MHz}$, respectively, off from the operating frequency for 

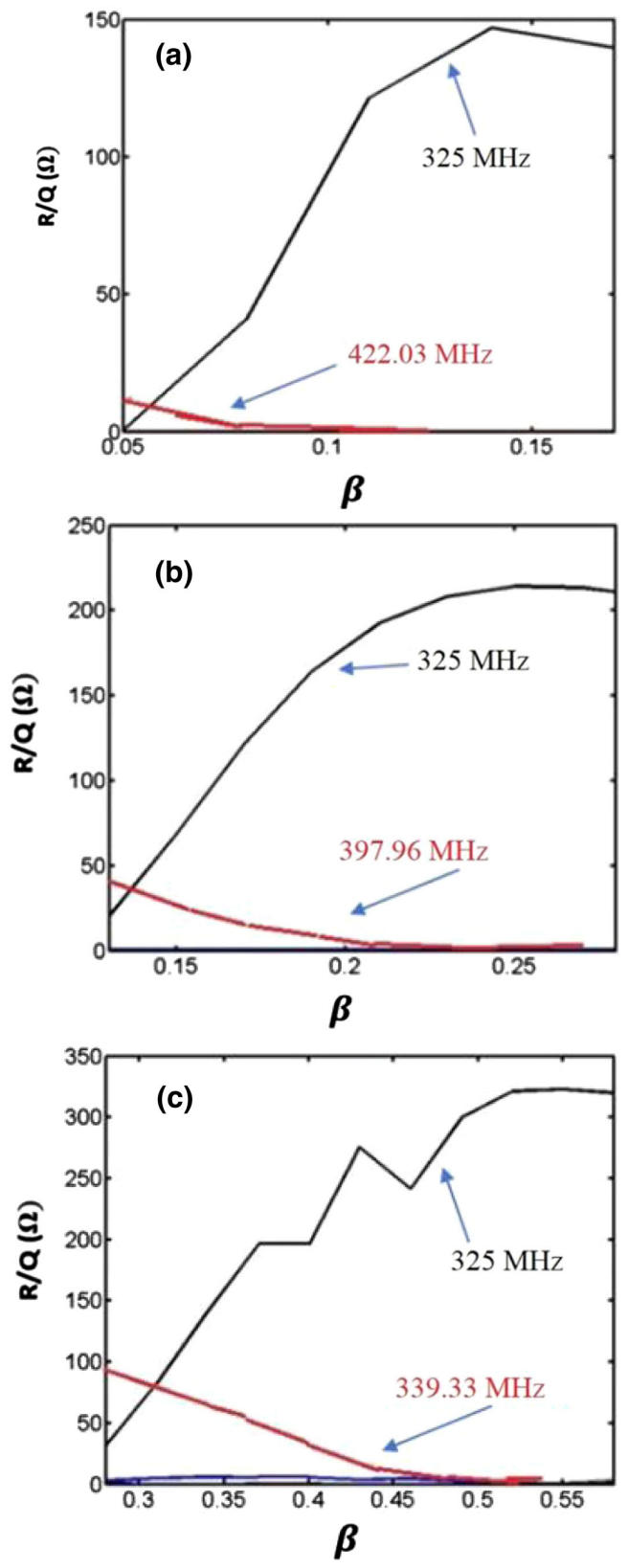

FIG. 21. Monopole modes R/Q for the (a) SSR-A, (b) SSR-B, and (c) SSR-C, cavities.

the three SSR cavities and so pose no threat in terms of the beam power coupling to these monopole modes. The R/Q of the dipole and quadrupole modes was calculated using Eq. (6) [32],

$$
\frac{R}{Q}=\frac{c^{2}}{\omega^{3} U a^{2}}\left|\int_{0}^{L} E_{z} e^{i k z} d z\right|^{2}
$$

where $a$ is the radial distance from the axis, and $U$ is the stored energy. The R/Q for these modes as a function of mode frequency is shown in Fig. 22; they can be seen to be less than $10^{-8}$, and should therefore not be a problem.
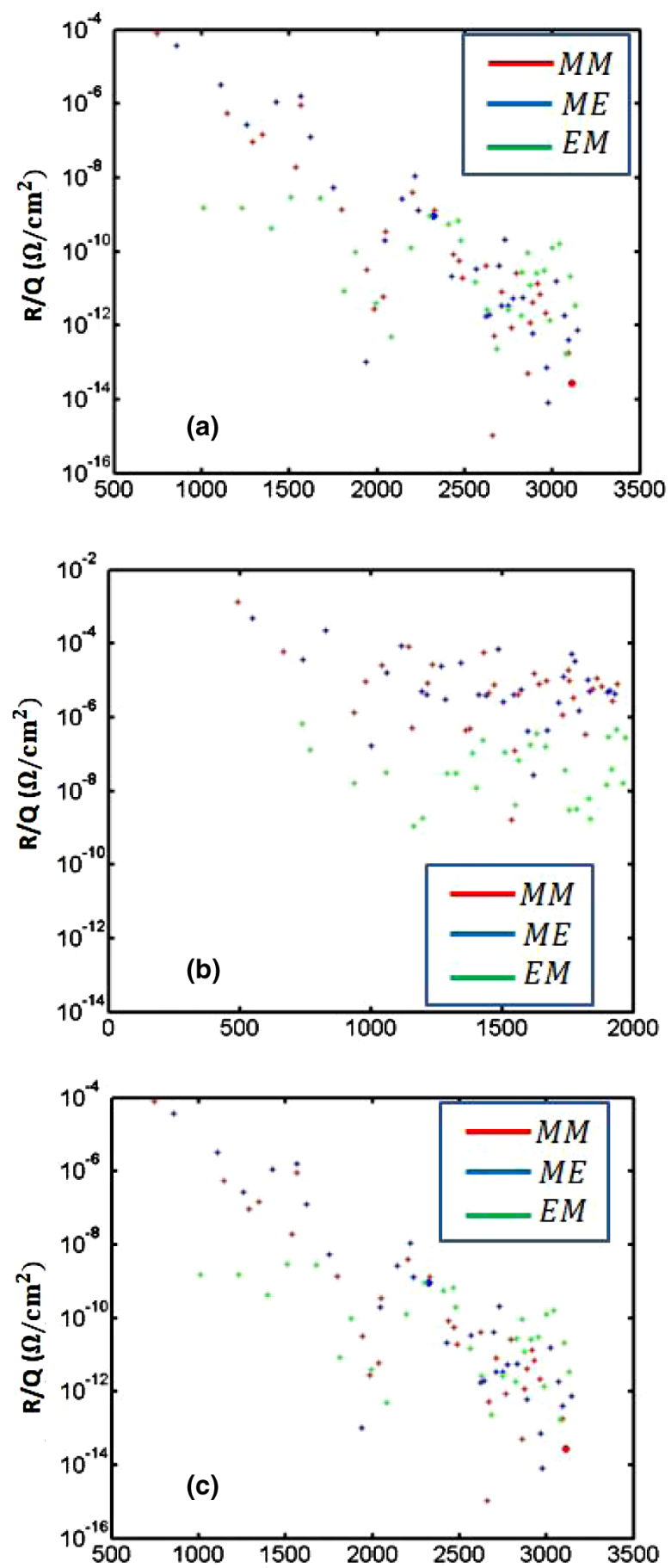

FIG. 22. Transverse R/Q for the dipole and quadrupole modes in the (a) SSR-A, (b) SSR-B, and (c) SSR-C, cavities.

\section{Multipacting}

Multipacting, which is the resonant amplification of secondary electron emission in the rf cavities, causes the absorption of rf power, making it impossible to increase the cavity fields even by raising the incident power. Besides, the electron impact on the structure walls causes the temperature to rise and eventually, in the case of superconducting cavities, to quenching. 

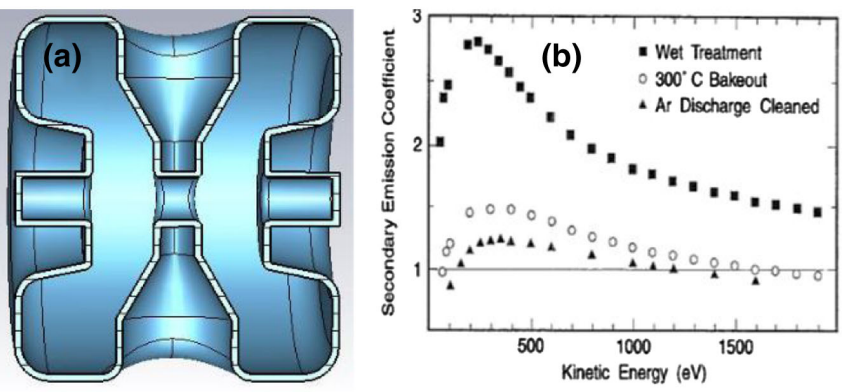

FIG. 23. (a) The shelled model for the SSR cavity and (b) the SEY plot for the three Niobium types that can be used to perform the multipacting simulations.

We performed multipacting simulation studies for the SSR-A, SSR-B, and SSR-C cavities. The multipacting simulations were performed with a shelled model of discharge cleaned Niobium SSR cavity, as shown in Fig. 23(a). One can also use the $300 \mathrm{deg}$ baked Niobium or wet treatment Niobium while dealing with a surface of different degrees of processing. The Secondary Electron Yield (SEY) plot [32] for all three Niobium types is shown in Fig. 23(b). With an increase in the surface quality, the time taken for initiation of multipacting increases, and therefore, the simulations need to be optimized in terms of the mesh cell, type of particle source, and simulation time. As the zone of multipacting was not known to start with, the simulations were performed with two different types of primary particle sources. In the first case, the particles are considered to be distributed in the cavity volume, as shown in Fig. 24(a), and for the second case, we chose all inner faces of the cavity as a particle source, as shown in Fig. 24(b). For both cases, the bunch parameters are listed in Table VI; these parameters are chosen after several iterations and are kept identical for both cases. In both cases the number of primary particles is prohibitively large, and though most of them die, initial calculations take an enormous amount of computational time. One of the ways to reduce such undesirable calculations is to specify the particle source in the multipacting prone zones only. Here we used the analytic formalism for
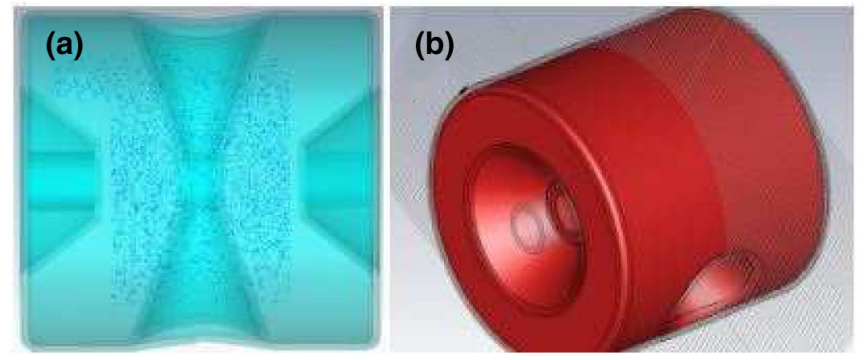

FIG. 24. Different types of primary particle source for the multipacting simulations: (a) particle volume source, and (b) whole cavity surface as a particle source.
TABLE VI. Electron beam parameters for the primary electron emission.

\begin{tabular}{lc}
\hline \hline Parameter & Value \\
\hline Emission Model & Gaussian \\
Particle type & Electron \\
Bunches & 1 \\
Sigma (ns) & 2 \\
Cutoff length (ns) & 3 \\
Kinetic energy (eV) & 4 \\
Kinetic spread (\%) & 100 \\
Angular spread (\%) & 100 \\
\hline \hline
\end{tabular}

two point multipacting [32], where the threshold field is given by,

$$
E=\frac{\omega^{2} d m}{\pi e},
$$

where $d$ is the separation between the surfaces of impact, $\omega$ is the angular frequency of the $\mathrm{rf}$ field, $e$ is the electronic charge, and $m$ is the electron mass.

The fields obtained from the rf simulations and Eq. (3.5) were used to get an approximate estimate of the multipacting zones, and the results are shown in Fig. 25. The electron trajectories obtained from the calculations suggest that the multipacting zones are localized and lie where the cavity wall meets the end wall and at the point where the spoke base meets the cavity wall. With this understanding of the secondary electron trajectories, we chose to define the particle source only in the regions that are prone to multipacting, allowing us to reduce the number of particles in the simulations, making it less time-consuming. The particle source chosen for our simulation is shown in Fig. 26. To ensure that the results obtained with all three types of particle sources are the same, we plotted the particle data as a function of time for all three cases, and the results are shown in Fig. 27.

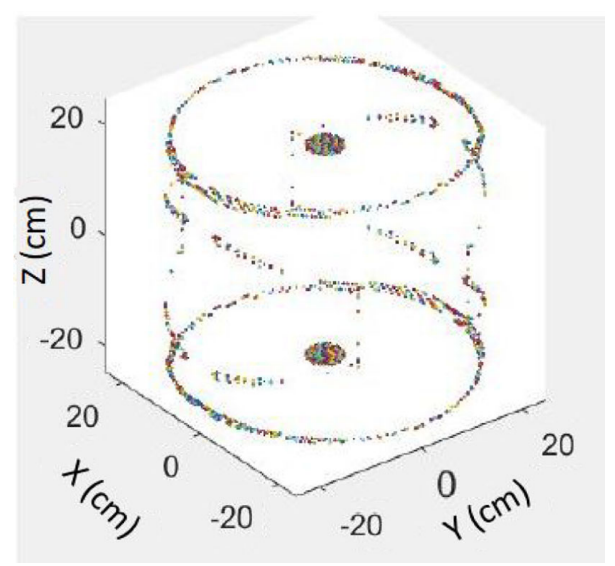

FIG. 25. Multipacting sites calculated from the exported rf fields and the condition for the two-point multipacting. 


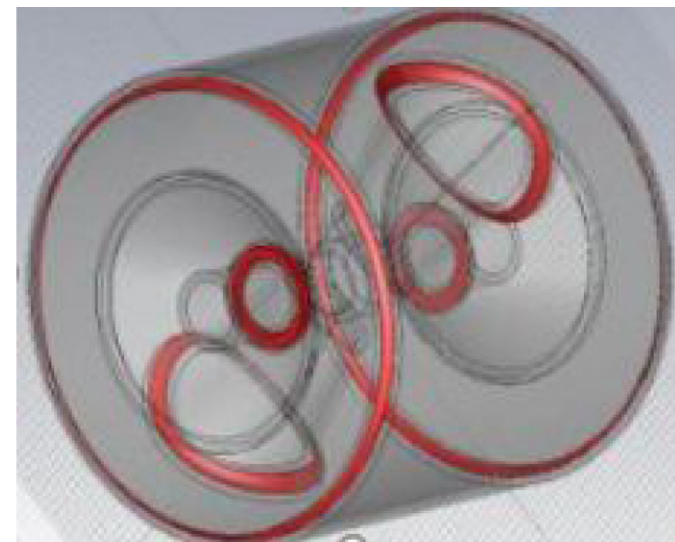

FIG. 26. The particle source defined in the zones of multipacting.

Figure 27 shows that, for all practical purposes, the results obtained using all three particle sources are identical and therefore we chose to use the third option where the primary particles originate only in the multipacting prone zones as shown in Fig. 25.

The PIC module that is adopted for the multipacting studies is pretty sensitive to the computational grid density. For models that are of the order of $50 \mathrm{~cm} X 50 \mathrm{~cm} \mathrm{X} 50 \mathrm{~cm}$, approximating the model with desired accuracy demand 100s of millions of mesh cells, which may exceed the capabilities of the computing resources available to us. Therefore we decided to exploit the one-eighth symmetry [40] of the model, as shown in Fig. 28. The open faces of the cavities are covered with a material with no secondary electron yield so that we do not consider any contribution from those surfaces; also, the metal acts as a fully reflecting surface for the particles and avoids any particle loss from those surfaces.

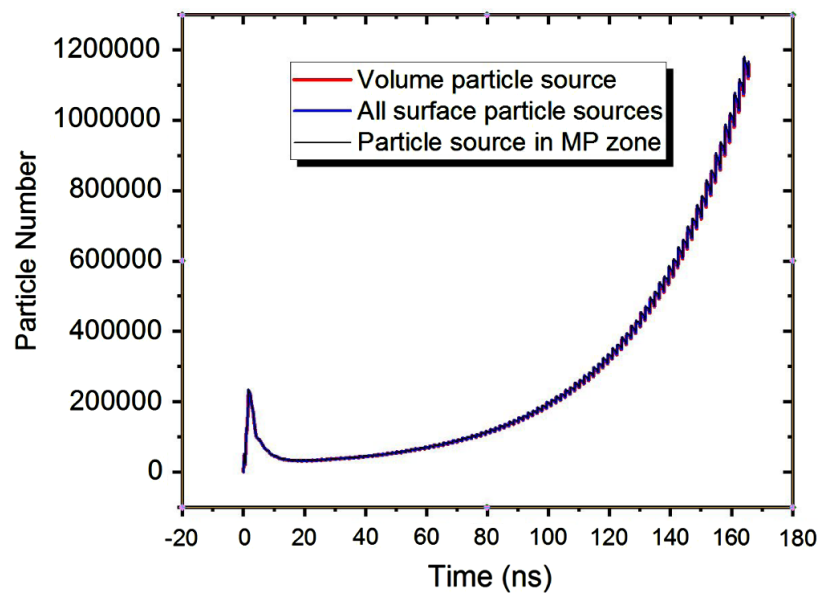

FIG. 27. Variation of the number of secondary electrons in the SSR-C cavity as a function of time, for three different types of primary particle sources.

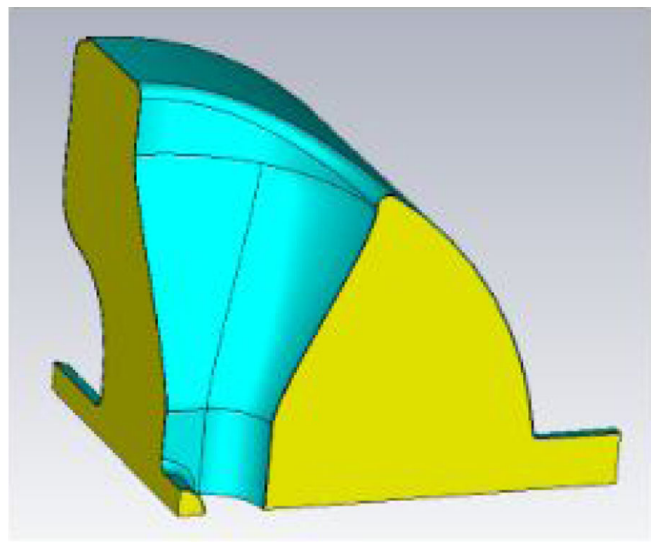

FIG. 28. One-eighth cavity structure used for the multipacting simulations.

Multipacting simulations were performed with the oneeighth model, as shown in Fig. 28 by varying the number of hexahedral mesh cells to obtain stable multipacting growth rate $(\alpha)$. The variation of $\alpha$ with the number of mesh cells is shown in Fig. 29. Our convergence analysis shows that for SSR-C the minimum number of required mesh cells is over 50 million. This growth rate convergence study was performed for the SSR-C structure, and we assume that SSR-A and SSR-B will also demonstrate convergence for this mesh density as their dimensions are smaller than SSR-C.

\section{Simulation results}

Once the stability of the simulation parameters was assured, multipacting studies were performed for SSR-A, SSR-B, and SSR-C with the imported rf fields and with different gradients.

The growth rate for the multipacting electron yield was calculated using the time average of the collision and emission currents from the cavity surface and is defined as,

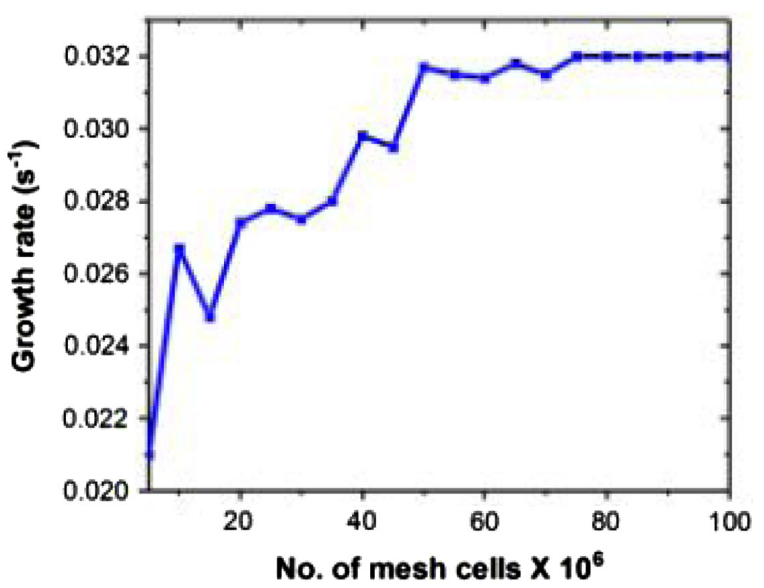

FIG. 29. Variation of the multipacting growth rate as a function of the number of the computational cells. 

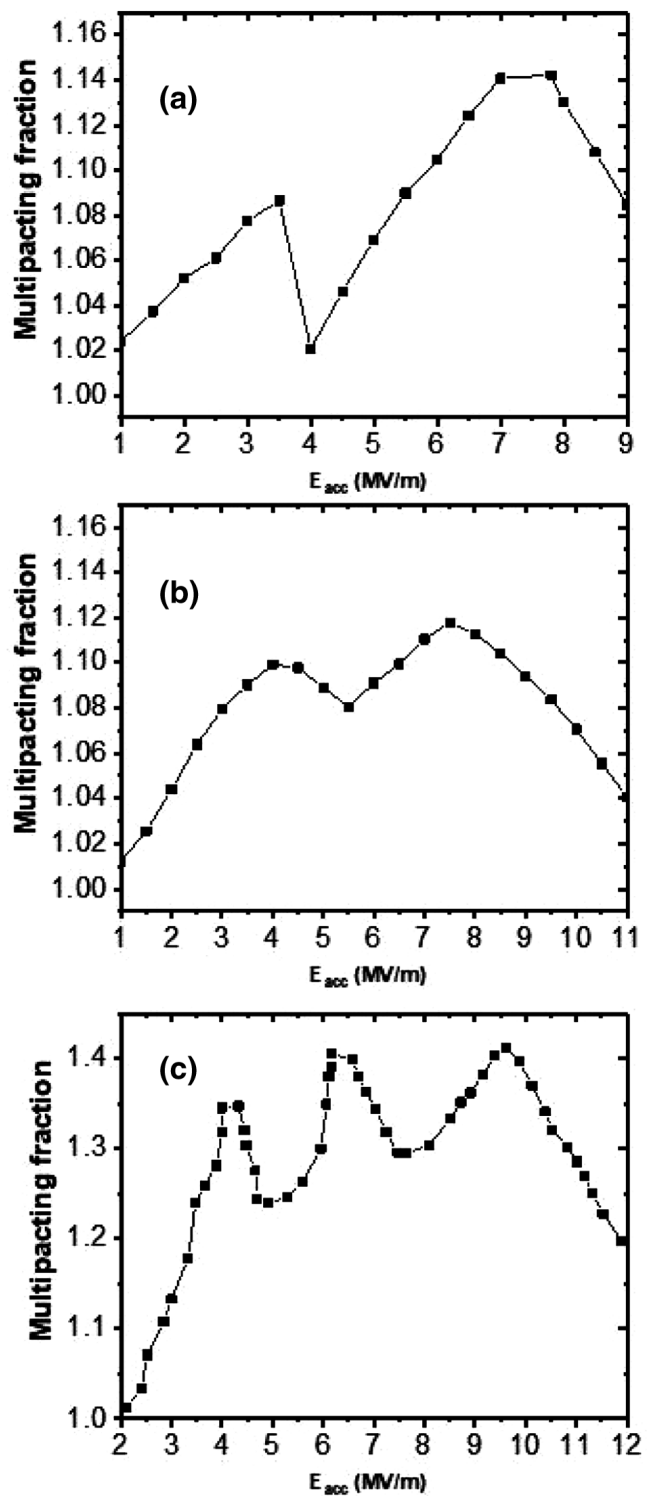

FIG. 30. Multipacting yield as a function of accelerating gradient for (a) SSR-A, (b) SSR-B, and (c) SSR-C.

$$
\alpha=\frac{1}{T_{\mathrm{RF}}} \ln \left(\frac{\int_{T 1}^{T 2} I_{\text {emission }} d t}{\int_{T 1}^{T 2} I_{\text {collision }} d t}\right) .
$$

The values for the secondary electron yield as a function of the accelerating gradient for SSRs are shown in Fig. 30. For SSR-A, the multipacting yield (MPY) varies between 1.02 to 1.14 , with two barriers at $3.5 \mathrm{MV} / \mathrm{m}$ and $7 \mathrm{MV} / \mathrm{m}$. For SSR-B, the MPY is lower than SSR-A with a minimum value of 1.02 at $1 \mathrm{MV} / \mathrm{m}$ and a maximum of 1.11 at $8 \mathrm{MV} / \mathrm{m}$. SSR-B also exhibits two multipacting barriers peaking at $4 \mathrm{MV} / \mathrm{m}$ and $8 \mathrm{MV} / \mathrm{m}$, respectively. The multipacting was observed to be most severe in the case of the SSR-C cavity, where the value of the MPY varies between 1 and 1.4 with three MP barriers at $4 \mathrm{MV} / \mathrm{m}$, $6 \mathrm{MV} / \mathrm{m}$ and $9 \mathrm{MV} / \mathrm{m}$.

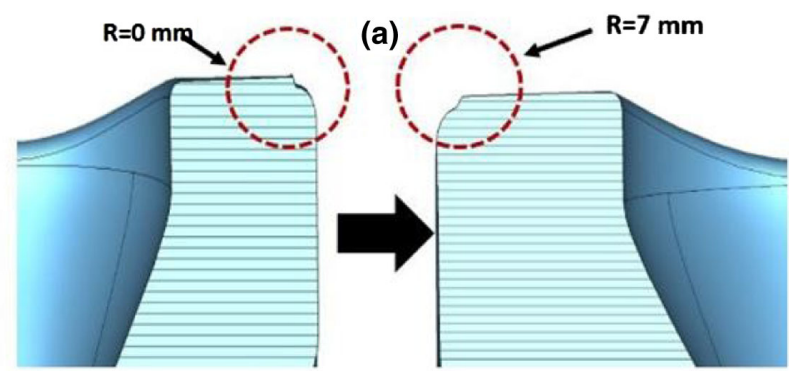

(b)
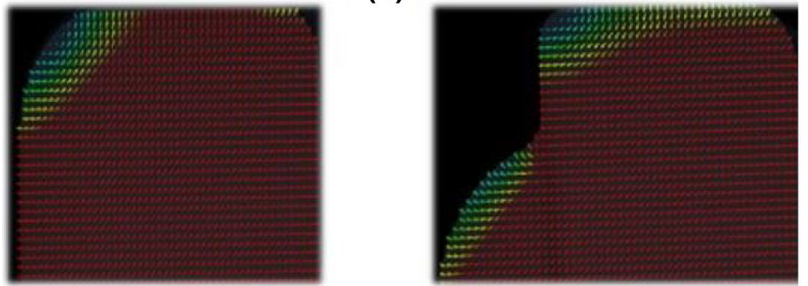

FIG. 31. Design modification in the SSR structure: (a) introduction of the double corner radius and (b) vector field plot in the multipacting zone before and after the introduction of the double corner radius.

As the MPY was observed to be higher in the SSR-C cavity, attempts were made to lower the secondary electron yield via geometric modifications in the cavity, as discussed in the next sub-section.

\section{Multipacting mitigation}

Since the multipacting growth rate for the SSR-C cavity was observed to be significant with the present cavity geometry, it may take several hours to condition [40] the cavity successfully. As the SSR-C cavity is dominated by 2-point multipacting, the cavity geometry at the multipacting zones was modified so that the symmetry for the two-point multipacting can be broken.

Here, following Ref. [40], we have introduced a double corner radius that reduces the tangential electric field component that was responsible for the two-point trajectory in that region. Figure 31(a) shows the introduction of the double corner radius from model 1.0 to model 2.0. Figure 31(b) shows the electric field in the multipacting zone. It is evident from the electric field vector plot that introduction of the double corner radius has reduced the components of the fields supporting the two-point multipacting. Figure 32 shows the comparison of the secondary electron yield without (model 1.0) and with (model 2.0) the double corner radius. It clearly shows a $14 \%$ reduction in the multipacting yield. Also, we could eliminate the soft barriers below $4 \mathrm{MV} / \mathrm{m}$.

\section{BEAM DYNAMICS}

In order to perform realistic beam dynamics simulations, three-dimensional fields from the optimized rf design of the spoke cavity and the magneto-static design of the 


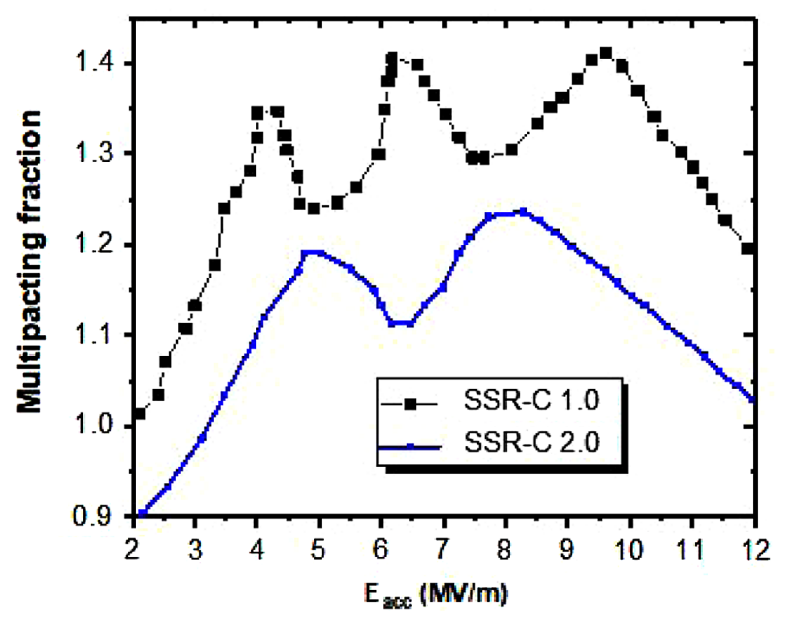

FIG. 32. Multipacting yield as a function of accelerating gradient before (model-1) and after (model-2) introduction of the double corner radius.

superconducting solenoid were imported from CST Microwave Studio for the lattice design and beam dynamics simulations. In the design of the SC section of the linac, primary emphasis has been given to the minimization of

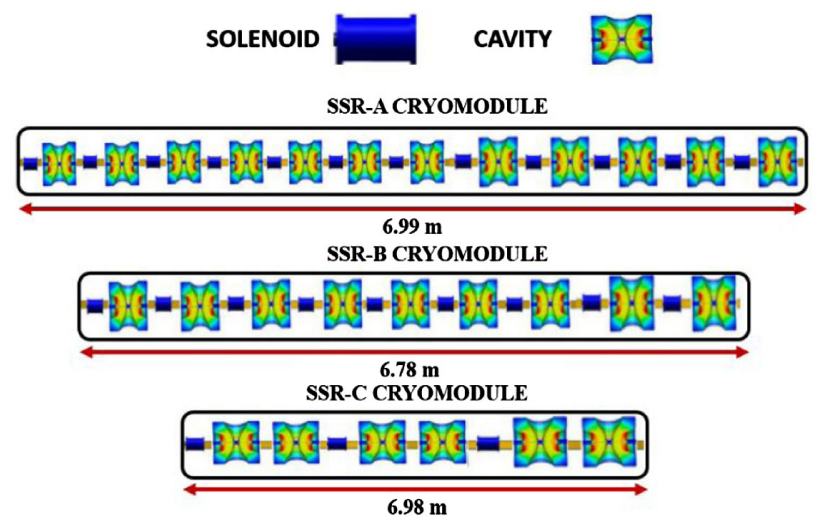

FIG. 33. Cryomodules for the three SSR sections.

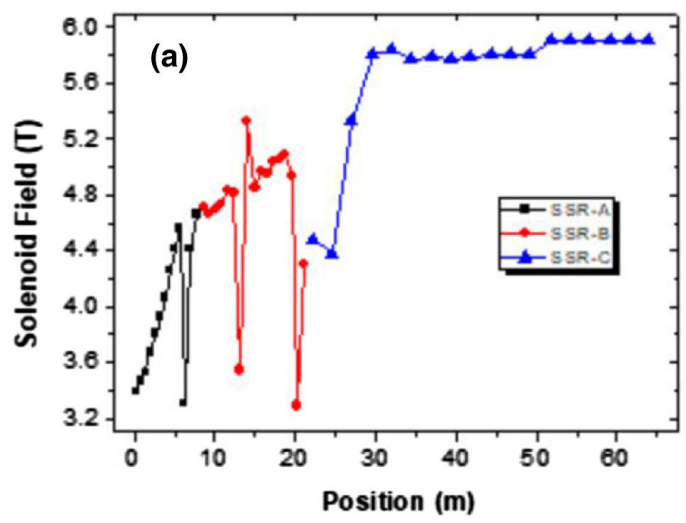

emittance growth and beam halo development while keeping the linac compact and cost-efficient.

As discussed by Struckmeier and Reiser [41], the zerocurrent phase advances were kept below $90^{\circ}$ to avoid the envelope instability due to any mismatch in the lattice. The operating point for the period operations was chosen in the resonant free region of the Hofmann chart [42] to avoid the parametric resonances and space-charge induced collective instability in the transverse and longitudinal planes. Considering the maximum extent of the beam halo proposed by Wangler [12], the aperture was chosen to be larger than six times the rms beam size.

The proposed lattice period consists of one single spoke cavity, a superconducting solenoid, and intermediate drifts for the SSR-A and SSR-B sections of the linac, where the solenoid is positioned after every cavity. For the SSR-C section of the linac, the lattice period is formed by two spoke cavities followed by a superconducting solenoid. All these lattices will be housed in a cryomodule; therefore, mechanical and thermal constraints need to be taken into account. Considering several insulation layers of the cryomodule and intermediate beam diagnostics, a separation of $400 \mathrm{~mm}$ was ensured between the cryomodules. Figure 33 shows the schematic of the cryomodule with the placement of the spoke cavity SC solenoid and drift spaces for SSR-A, SSR-B, and SSR-C sections of the MEHIPA linac.

The transverse and longitudinal fields of the lattice were tuned to satisfy the constraints on the structure phase advance $\left(\leq 90^{\circ}\right)$ and maximum operating gradient of the cavities $(8 \mathrm{MV} / \mathrm{m}, 10 \mathrm{MV} / \mathrm{m}$, and $11 \mathrm{MV} / \mathrm{m}$ for SSR-A, SSR-B, and SSR-C respectively). Figure 34 shows the variation of accelerating gradient and solenoid fields along the linac. For SSR-A the accelerating gradient ranges from 1.85 to $7.92 \mathrm{MV} / \mathrm{m}$, for SSR-B from 2.84 to $10.03 \mathrm{MV} / \mathrm{m}$, and for SSR-C from 4.84 to $10.82 \mathrm{MV} / \mathrm{m}$. As shown in Fig. 34 accelerating fields of the spoke cavities were ramped slowly and were kept constant to maintain the phase advance criterion. Also, the lower limit of the field

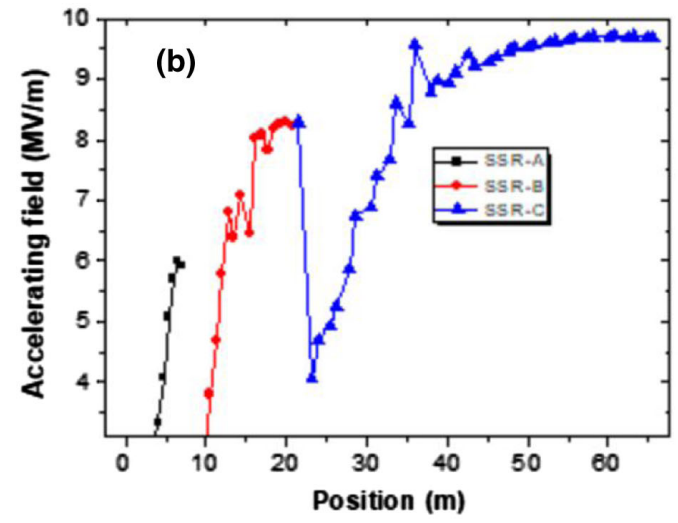

FIG. 34. (a) Magnetic fields of the superconducting solenoids in the superconducting section of the MEHIPA linac, and (b) cavity accelerating field calculated over the effective cavity length $\left(\beta_{\mathrm{opt}} \lambda\right)$ along the SC section of the MEHIPA linac. 

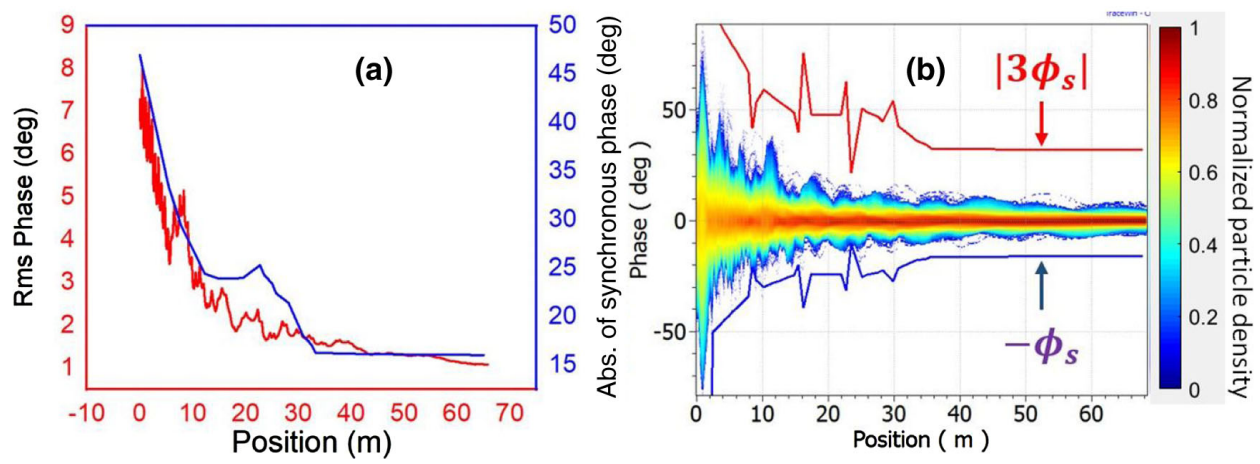

FIG. 35. (a) Variation of rms beam phase and absolute synchronous phase along the linac, and (b) maximum beam phase variation superposed with phase acceptance along the linac.

was chosen above the multipacting threshold to avoid the occurrence of multipacting in the cavities. For the superconducting solenoids, the maximum field was kept below $6 \mathrm{~T}[34,35]$. In the SSR-A lattice the required solenoid field varies between 3.4 and $4.57 \mathrm{~T}$, in the SSR-B lattice between 3.44 and $5.51 \mathrm{~T}$, and in the SSR-C lattice between 3.26 and $5.90 \mathrm{~T}$.

With the accelerating gradient shown in Fig. 34 and for the given energy ranges of 3-10 MeV, $10-40 \mathrm{MeV}$ and 40-200 MeV, the longitudinal beam dynamics demands 12 SSR-A cavities, 18 SSR-B cavities, and 36 SSR-C cavities. The synchronous phase has been ramped linearly from -47 to -30 , from -30 to -20 and from -20 to -15 for SSR-A, SSR-B, and SSR-C respectively. The synchronous phase was chosen such that the beam bunch is well within the phase acceptance of the spoke cavities while keeping the total number of cavities minimum. Figure 35 shows the variation of the absolute value of the synchronous phase and beam phase along the linac.

As shown in Fig. 35, the beam phase width decreases from $80 \mathrm{deg}$ to $20 \mathrm{deg}$ along the linac because of phase damping with acceleration. The synchronous phase along the linac was chosen, such that the phase acceptance is maximum at low energy and decreases with energy, thus ramping-up the acceleration rate. The phase acceptance varies from $140 \mathrm{deg}$ at the beginning of SSR-A to $48 \mathrm{deg}$ in the SSR-C section of the linac. This choice of synchronous phase ensures efficient phase focusing at low energy where the space-charge forces are higher.

With the obtained number of cavities, it was decided to place them in one, two, and six cryomodules for SSR-A, SSR-B, and SSR-C respectively. Other lattice details are listed in Table VII.

A fully three-dimensional PIC beam dynamics simulation was conducted with the lattice detailed above for the superconducting section of MEHIPA. The SSR-A section was injected with a $3 \mathrm{MeV}$ matched proton beam from the MEBT and was accelerated through all three families (SSRA, SSR-B, and SSR-C) of spoke resonators. The intercryomodule spacing of $400 \mathrm{~mm}$, devoid of longitudinal and transverse focusing, has a detrimental effect on the beam dynamics in terms of emittance and halo increase. The cavity and solenoid fields were scanned to obtain the minimum emittance and halo parameters at the exit of the linac. Then, keeping in consideration the constraints due to longitudinal and transverse phase advance and

TABLE VII. Parameters for the superconducting section of MEHIPA.

\begin{tabular}{lccc}
\hline \hline Parameter & SSR-A & SSR-B & SSR-C \\
\hline Energy Range (MeV) & $3-9.13$ & $9.13-40.50$ & $40.50-206.42$ \\
Synchronous Phase (deg) & $-47^{\circ}$ to $-30^{\circ}$ & $-30^{\circ}$ to $-21^{\circ}$ & $-21^{\circ}$ to $-16^{\circ}$ \\
No. of cavities & 12 & 18 & 36 \\
Cavity length (mm) & 176 & 286 & 560 \\
$\beta_{\text {opt }}\left(\beta_{\text {opt }} \lambda\right)(\mathrm{mm})$ & 0.144 & 0.256 & 234 \\
$L_{\text {eff }}$ & 113 & 10 & 491 \\
Accelerating gradient $(\mathrm{MV} / \mathrm{m})$ & 8 & 30 & 11 \\
Beam tube diameter $(\mathrm{mm})$ & 30 & 250 & 40 \\
Solenoid field map length $(\mathrm{mm})$ & 250 & $4.6-5$ & 320 \\
Solenoid field (T) & $3.4-4.6$ & 108.92 & 108.92 \\
Solenoid to cavity length (mm) & 77.77 & $\ldots$ & 352.29 \\
Cav. to cryostat (mm) & 77.77 & $\ldots$ & 352.29 \\
Cav. to Cav. (mm) & $\ldots$ & 184.58 \\
\hline \hline
\end{tabular}



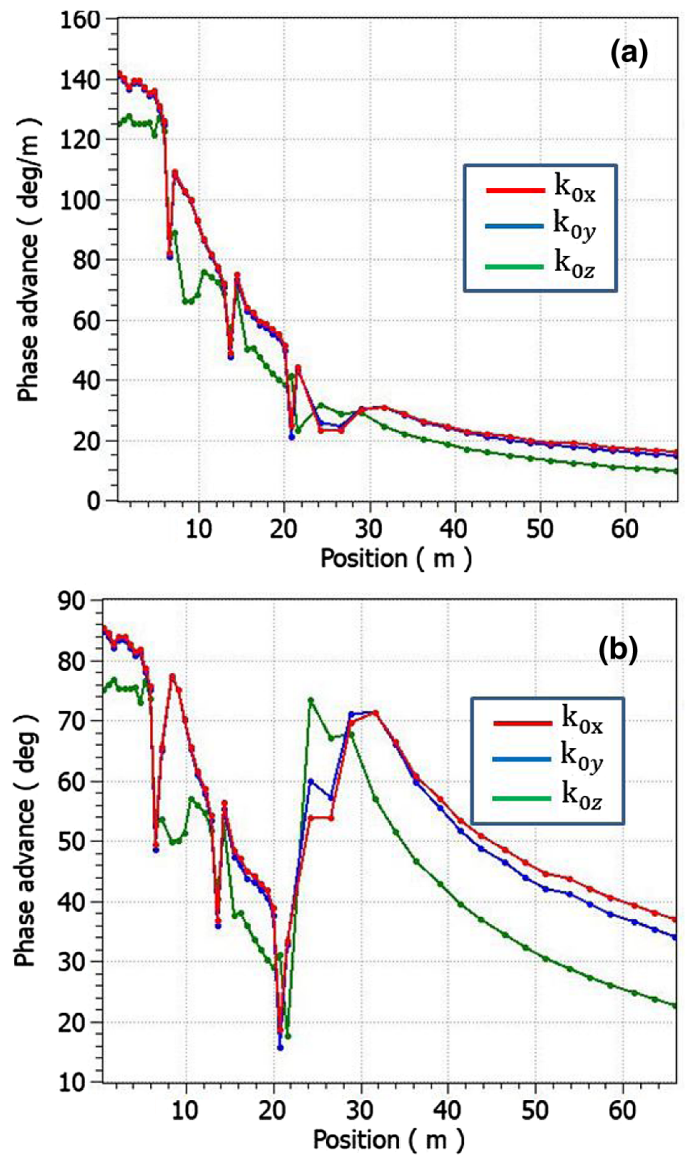

FIG. 36. (a) Variation of zero-current phase advance per unit length along the SC section of the MEHIPA linac, and (b) variation of the zero-current phase advance per period along the SC section of the MEHIPA linac. maximum cavity and solenoid fields, we choose the cavity and solenoid fields that minimize the emittance growth.

The intersection beam matching during the transport was performed using the last and penultimate solenoids and cavity synchronous phase of a given spoke family, while maintaining the required energy gain and phase advances. The intersection matching disrupts the smooth variation in the structure phase advance per unit length but is an essential step to avoid emittance growth, halo formation and beam loss in the linac. Figure 36 shows the variation of the structure phase advance per unit length and structure phase advance per period along the SC section of the MEHIPA linac. As Fig. 36 shows, throughout the linac the structure phase advance per period remains below $90^{\circ}$, and apart from the fluctuations introduced because of the intrasection matching, the variation in the phase advance shows a smooth transition.

The beam quality along the linac was monitored through the rms beam size, rms normalized transverse and longitudinal emittances, and the transverse and longitudinal halo parameters that are defined as a ratio of the fourth moment of the distribution to the second moment of the distribution. Figure 37, and Fig. 38 summarize the behavior of the beam along the superconducting linac.

Figure 37(a) demonstrate that the beam is well-matched with an average beam size of $1.8 \mathrm{~mm}$ and the maximum rms beam radius $(2 \mathrm{~mm})$ is less than six times the beam aperture $(20 \mathrm{~mm})$. The beam size suffers slight blow-up in the drift that separates the cryomodules because of lack of focusing elements. At the end of the SC section of the linac, we obtained a beam that is $1.4 \mathrm{~mm}$ in the $\mathrm{x}$-direction and $1.2 \mathrm{~mm}$ in the y-direction. (a)
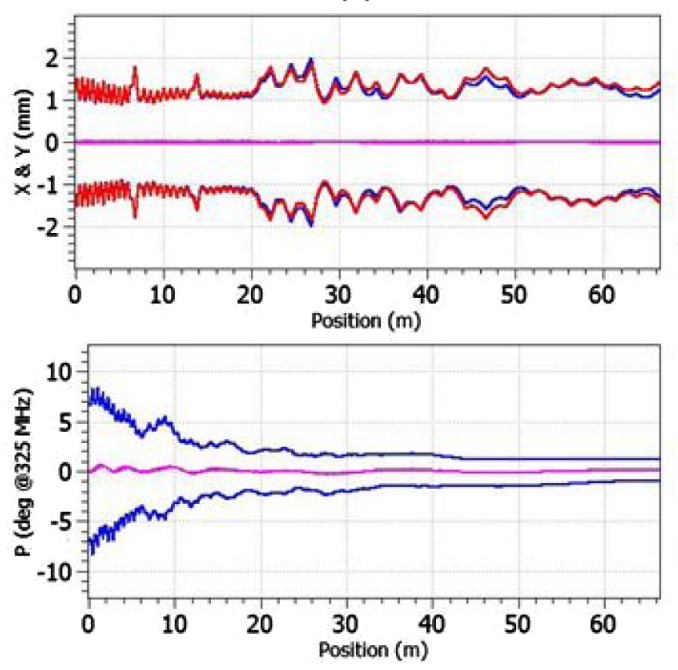

(b)
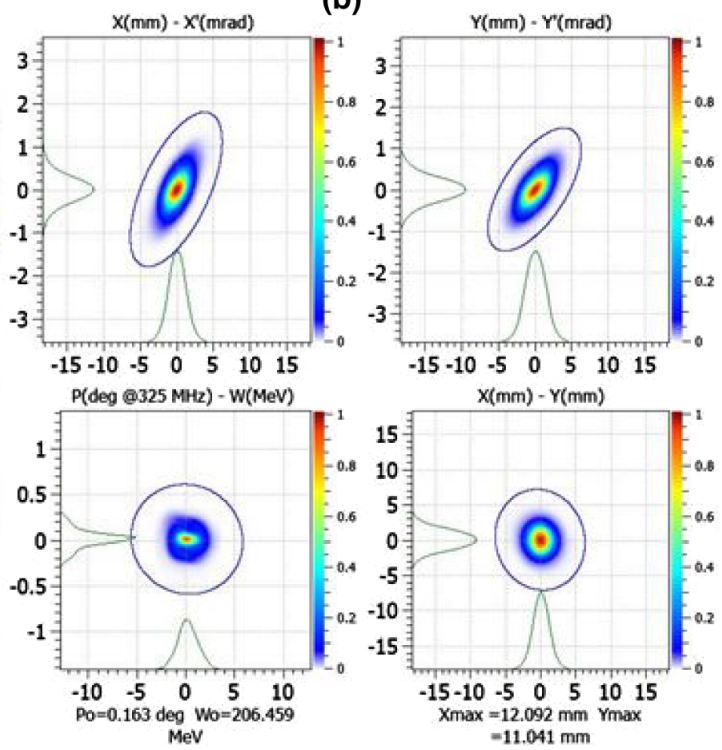

FIG. 37. (a) Evolution of transverse and longitudinal RMS beam size, and (b) Phase-space and coordinate-space particle density distribution at the exit of the SC section of the MEHIPA linac. 

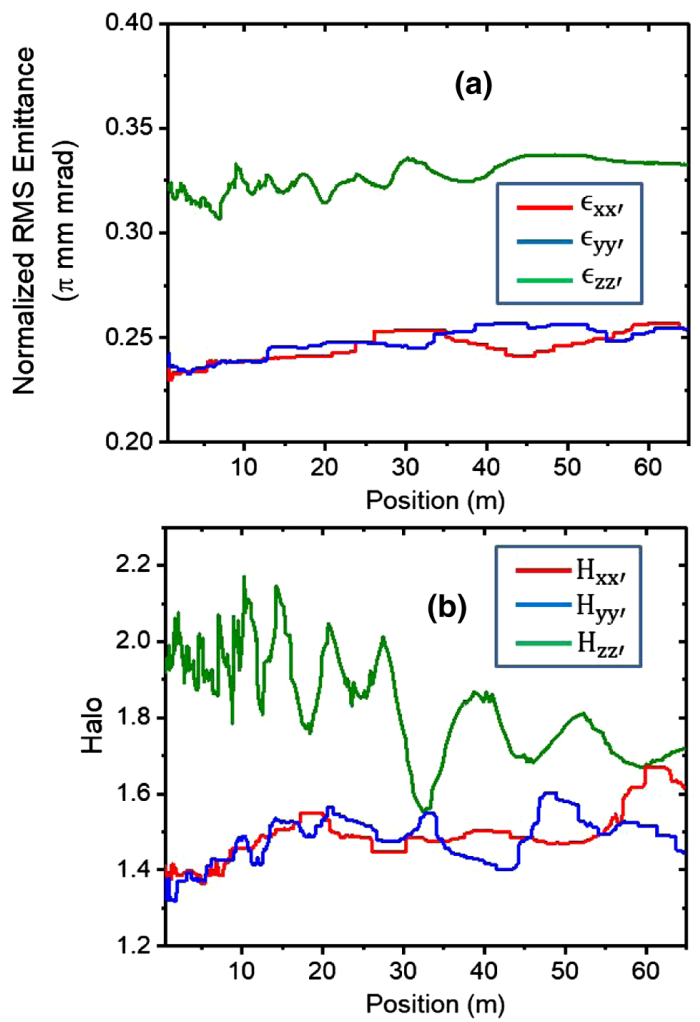

FIG. 38. (a) Transverse and longitudinal rms normalized emittance, and (b) transverse and longitudinal beam halo parameter along the SC section of the MEHIPA linac.

The beam size gives a quantitative estimate of the beam aperture but does not render any estimate of the beam quality; therefore, we inspected the evolution of the beam phase-space in terms of its second and fourth moments. The second moment that is the beam emittance is shown in Fig. 38(a), and the beam halo parameter that is defined as a ratio of the fourth moment to the square of the second moment is shown in Fig. 38(b). As shown in Fig. 38(a) the beam suffers an emittance growth of $9.2 \%, 11.1 \%$, and $4.7 \%$ in the $\mathrm{x}-\mathrm{x}^{\prime}, \mathrm{y}-\mathrm{y}^{\prime}$, and $\mathrm{z}-\mathrm{z}$ ' planes, respectively. The emittances after each section of the linac are listed in Table VIII. The halo parameter demonstrates similar behavior with an increase of $17 \%, 10 \%$, and $-8.5 \%$ in the $x-x^{\prime}, y-y^{\prime}$, and $\mathrm{z}-\mathrm{z}$ ' planes, respectively.

As mentioned earlier, the SC section of MEHIPA adopts solenoid focusing and should therefore provide an axisymmetric beam, but, as shown in Fig. 38, the emittance and halo parameter show an asymmetric behavior. One of the plausible reasons for such behavior is the transverse asymmetry introduced by the spoke. To investigate the contribution from the spoke cavities toward the asymmetric behavior of the beam, the change in the transverse divergence was calculated using the Lorentz force equation without consideration of space-charge forces and the electric and magnetic field components of the cavity at
TABLE VIII. Beam parameters at the exit of the superconducting section of MEHIPA linac.

\begin{tabular}{llll}
\hline \hline Paramters & SSR-A & SSR-B & SSR-C \\
\hline$\epsilon_{x}(\pi \mathrm{mm}-\mathrm{mrad})$ & 0.244 & 0.252 & 0.251 \\
$\epsilon_{y}(\pi \mathrm{mm}-\mathrm{mrad})$ & 0.243 & 0.260 & 0.252 \\
$\epsilon_{z}(\pi \mathrm{mm}-\mathrm{mrad})$ & 0.328 & 0.316 & 0.332 \\
Halo $_{X}$ & 1.44 & 1.42 & 1.60 \\
Halo $_{Y}$ & 1.41 & 1.44 & 1.43 \\
Halo $_{Z}$ & 2.20 & 2.01 & 1.72 \\
\hline \hline
\end{tabular}

the rms beam size. Figure 39 shows the calculated divergence along the linac because of the spoke cavities.

Figure 39 shows that the divergence kicks imparted by the rf field of the spoke cavity are asymmetric, and as the cavity length increases from SSR-A to SSR-C, the effect becomes more significant. This study demonstrates higher average Lorentz forces because of rf fields in the $\mathrm{x}$-direction than in the $\mathrm{y}$-direction, leading to a larger beam size in the x-direction than in the y-direction. The asymmetry was observed to be maximum toward the end of the SSR-C section with an average difference of $0.03 \mathrm{mrad}$ in the $\mathrm{x}$ - and $\mathrm{y}$-directions. This difference in the divergences leads to a difference in the beam size and also contributes to an asymmetric emittance growth in the transverse plane. We performed design changes suggested in [43] to reduce the asymmetry introduced because of the spoke but observed an increase in the peak electric field ratio. As the observed asymmetry is not significant and does not lead to any unacceptable beam quality deterioration, we accept this minor beam asymmetry in the linac.

Apart from the contribution from the rf fields, another vital factor that can cause beam deterioration is the space charge induced collective instability proposed by I. Hofmann. As shown in Ref. [44] the stability-chart analysis

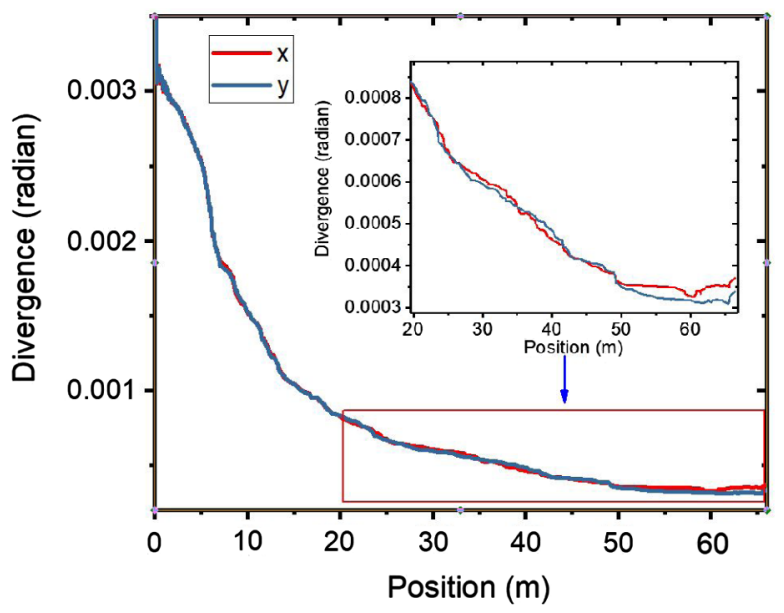

FIG. 39. Asymmetric divergence imparted to the beam by the rf field of the spoke cavity along the SC section of the MEHIPA linac. 


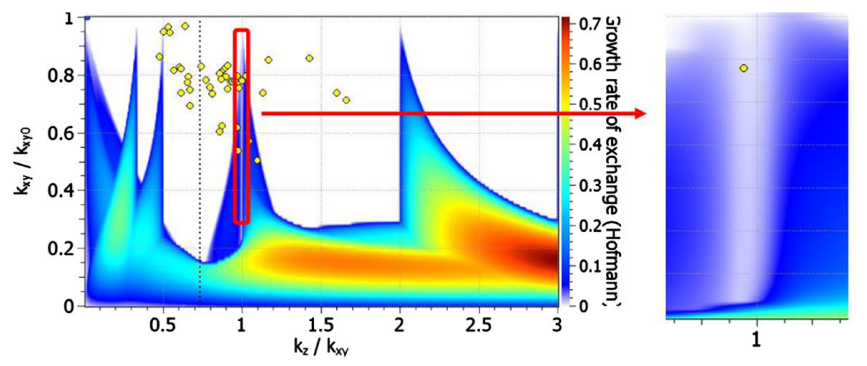

FIG. 40. Stability chart representing the second, third, and fourth-order resonances in the linac overlapped with the period operating points.

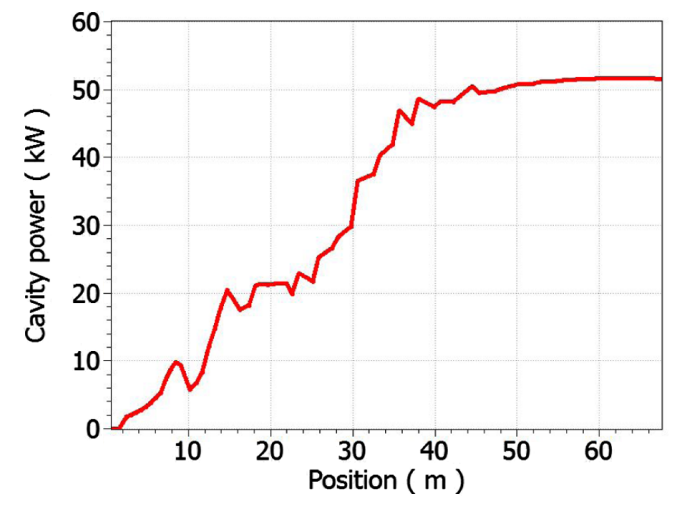

FIG. 41. Cavity power along the MEHIPA linac.

is an essential endeavor for the space-charge dominated beam, and therefore we plotted the Hofmann chart using the code TraceWin, shown in Fig. 40.

The stability chart clearly demonstrates the nonresonant operation of the linac. We do observe fast crossing of seven lattice periods with the tune depression more than 0.4 and therefore has no significant effect on the beam dynamics. The crossing of the phase advances shown in the tune footprint is because of the intrasection matching that was performed using the spoke cavity and the solenoid in the lattice.

\section{POWER REQUIREMENT AND CRYOGENIC LOAD}

The rf power delivered to the cavity has two components: the beam power and the power dissipated in the structure. The structure losses are significant in the normal conducting RFQ but are a negligible fraction of the total power for the superconducting cavities. The power generation part consists of an rf power amplifier and its high voltage power supply. For high energy particle accelerators, the conventional rf power amplifier is the klystron, and a high power CW klystron will be used to power the RFQ. The SC section of MEHIPA demands $66 \mathrm{rf}$ systems, and it has been planned to use one solid-state amplifier per cavity. The power requirement per cavity along the superconducting linac is shown in Fig. 41. It accounts for the power loss in transmission (assuming cable for $325 \mathrm{MHz}$ and waveguide for $650 \mathrm{MHz}$ ) and the power margin required for the effective operation of the voltage control system.

The rf power margin was chosen to be sufficient to control rf phase noise induced by microphonics (mainly related to helium pressure fluctuations) without a fast tuner at the nominal cavity loaded bandwidth of $66 \mathrm{~Hz}$. A power margin of $80 \%$ has been taken [10] to account for the transmission loss and the power required for the effective operation of the low-level rf (LLRF) voltage control system. The requirements of the rf amplifiers, taking into account this margin, are given in Table IX.

The power margin is a conservative one and has been taken keeping in mind our experience with LEHIPA. Note that this margin is used only for estimating the requirement from the rf source, and does not affect the linac design. As and when we develop and test prototypes, we will be able to refine these numbers.

The cryostats housing the superconducting devices represent a significant fraction of the capital cost of the cryogenic system for MEHIPA. They also greatly influence operational costs through their thermal insulation performance, which determines heat loads on the system. The cryostats also perform essential functions as diverse as support and precise positioning of the superconducting devices, containment of cryogenic fluids under steady and transient operating modes and leak tightness of the insulation vacuum. The minimization of the total heat load is essential for the efficient performance of the system.

The total heat load of the cryomodules comprises static and dynamic heat loads. The static heat load accounts for the heat load on the cryogenic system when the cryomodule is fully installed and operating under nominal conditions, with the exception that no rf power is provided to the couplers, and no beam is present. Estimating typical values for heat flux at low temperature transported by radiation, gas, and solid conduction is necessary for efficient thermal

TABLE IX. MEHIPA high power rf requirements.

\begin{tabular}{lcccccc}
\hline \hline $\begin{array}{l}\text { Cavity } \\
\text { type }\end{array}$ & Freq.(MHz) & $\begin{array}{c}\text { No. of rf } \\
\text { cavities }\end{array}$ & $\begin{array}{c}\text { No. of rf amplifiers } \\
\text { per cavity }\end{array}$ & $\begin{array}{c}\text { Operating } \\
\text { regime }\end{array}$ & $\begin{array}{c}\text { Beam } \\
\text { power }(\mathrm{kW})\end{array}$ & $\begin{array}{c}\text { rf amplifier } \\
\text { power }(\mathrm{kW})\end{array}$ \\
\hline SSR-A & 325 & 12 & 1 & CW & 10 & 18 \\
SSR-B & 325 & 18 & 1 & CW & 22 & 39.6 \\
SSR-C & 325 & 36 & 1 & CW & 50 & 90 \\
\hline \hline
\end{tabular}


TABLE X. MEHIPA cryogenic requirement.

\begin{tabular}{lcccc}
\hline \hline CM Type & $\begin{array}{c}\text { Number } \\
\text { of CMs }\end{array}$ & $\begin{array}{c}\text { Dynamic } \\
\text { load per CM }(\mathrm{W})\end{array}$ & $\begin{array}{c}\text { Static load } \\
\text { per CM (W) }\end{array}$ & $\begin{array}{c}\text { Total load at } \\
\text { 2K per CM (W) }\end{array}$ \\
\hline SSR-A & 1 & 15.2 & 3.8 & 19 \\
TSR-B & 2 & 35.8 & 8.95 & 44.75 \\
at 2K (W)
\end{tabular}

design. In high energy accelerators with intense beams, the cryostat heat leaks represent only a fraction of the total heat load budget, and the cryogenic system must also be designed to cope with large dynamic loads. The dynamic heat load is due to the rf losses and beam losses in the cavity. Table X presents the heat load budget for the SSR cryomodules. Here the static heat load is taken to be $25 \%$ of the dynamic heat load. The thermal design for optimizing the static heat load needs to be done for an accurate estimate of these heat loads. An excellent thermal insulation design helps limit the static heat load to a small fraction of the total heat load. Note that for a CW accelerator (unlike a pulsed one), the dynamic heat load, arising from the Ohmic dissipation needed to sustain the required fields, is dominant. The quantitative estimate of the static load comes from discussions with the PIP-II group at Fermilab as they are also working on a similar linac [10] architecture.

\section{RELIABILITY ANALYSIS}

An indispensable component that needs to be considered while designing a complex system like a high-intensity proton accelerator is its reliability. A thorough reliability study enables us to design an accelerator with a better understanding of the impact of component failure on accelerator operation, and ways of compensating for these potential failures.

We performed a detailed study to investigate the linac operation in terms of beam quality and beam transmission in the presence of a cavity or solenoid failure. We performed this analysis in two separate stages. The first stage determines the deterioration in the beam quality in terms of emittance and beam transmission with cavity and solenoid failure. This analysis fixes the critical accelerating and focusing elements in the linac through a controlled deactivation of one component at a time.

Figures $42(\mathrm{a}-\mathrm{c})$ show the emittance growth and beam transmission at the exit of the linac, as a function of cavity
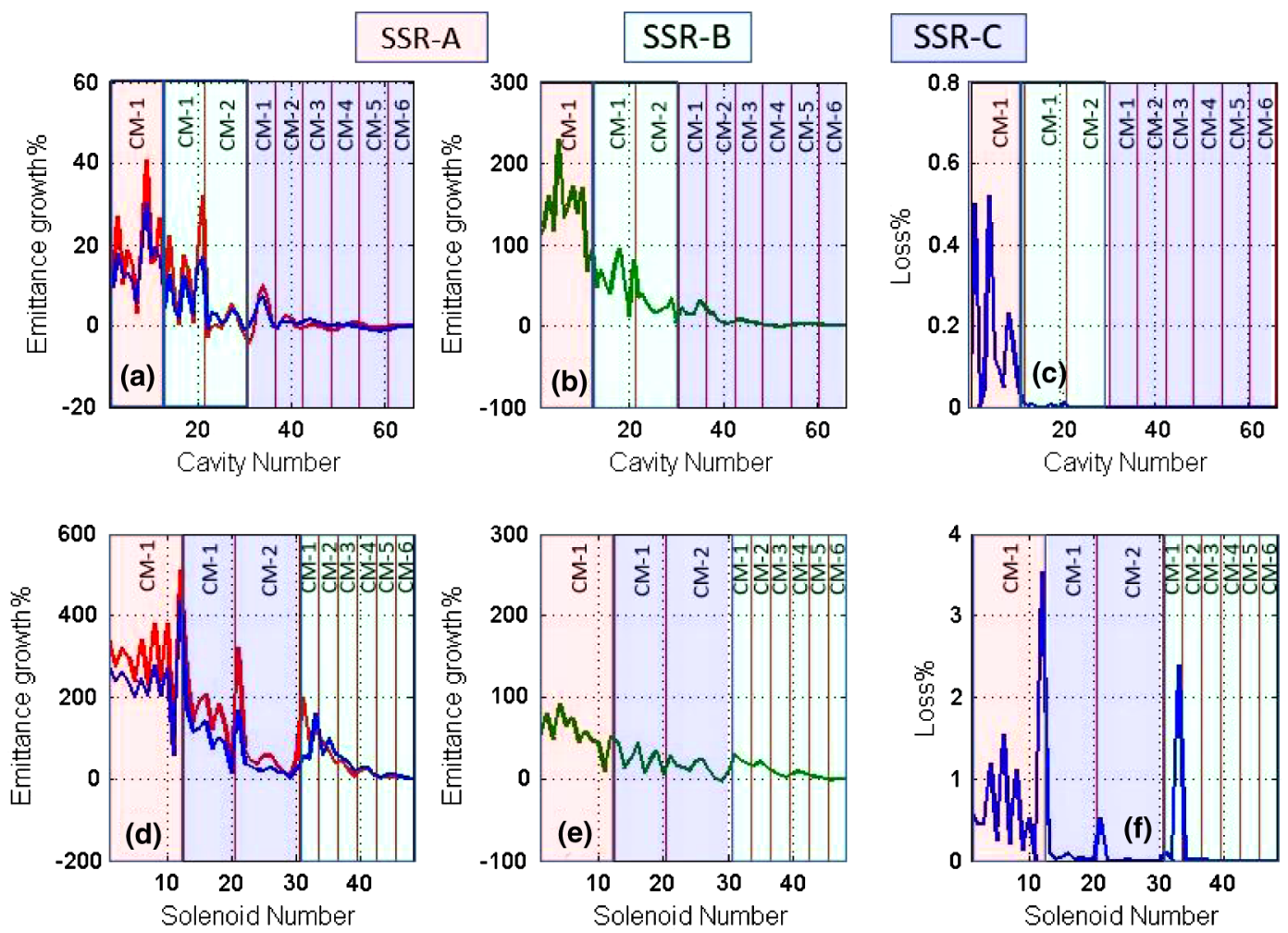

FIG. 42. Variation of (a) transverse emittance because of cavity failure, (b) longitudinal emittance because of cavity failure, (c) particle loss \% because of cavity failure, (d) transverse emittance because of solenoid failure, (e) longitudinal emittance because of solenoid failure, and (f) particle loss \% because of solenoid failure. 
failure (assuming just the one cavity fails). It can be seen that the spoke cavities' failure in the SSR-A section of the linac, where the space-charge forces are relatively higher, has maximum impact on the linac performance. However, the longitudinal phase-space suffers considerable emittance growth with a maximum value of $230 \%$ with the failure of the fifth spoke cavity. As the beam energy increases in the following sections of the linac, the cavity failure's influence decreases. In the SSR-B section of the linac, we observe a maximum increase of $100 \%$ in the longitudinal emittance when the fourth SSR-B cavity fails. The SSR-C section of the linac shows an insignificant effect on the longitudinal emittance.

The malfunctioning of these accelerating cavities also leads to transverse emittance growth. We observe the growth to be maximum in the SSR-A section with a maximum value of $40 \%$, followed by a maximum of $35 \%$ in the SSR-B section. The cavity failure in the SSR-C section of the linac has no critical role in the transverse emittance growth. Our analysis shows that the failure of the cavities in the proximity of the intercryomodule drift causes maximum emittance growth, as was observed for the seventh SSR-A cavity failure and ninth SSR-B cavity failure leading to an emittance growth of $40 \%$ and $35 \%$ respectively.
The observed significant deterioration of the beam quality in the longitudinal phase-space causes beam loss up to $0.5 \%$ in case of failure of the first and fifth SSR-A cavities. We do not observe any particle loss in the later section of the linac. Fig. 42(c) suggests that the failure of the first and the fifth SSR-A cavities is most critical and demands immediate retuning to avoid beam loss.

Next, we investigated the scenario where individual solenoids were deactivated, one at a time, and the beam quality and beam transmission were observed at the exit of the linac. Figures $42(\mathrm{~d}-\mathrm{f})$ show the impact of solenoid failure on the beam emittance and beam transmission. As a solenoid confines the beam in the transverse plane, its failure affects the beam trajectory transversely, leading to a higher transverse emittance growth followed by particle loss. As shown in Fig. 42(d), the last solenoid of SSR-A is most critical and causes a transverse emittance growth of $500 \%$ and a beam loss of $3.5 \%$ at the exit of the linac. Figures $42(\mathrm{~d}-\mathrm{f})$ also suggest that solenoids in the proximity of the intracryomodule drift are crucial and demand immediate attention to avoid particle loss during beam operation.

The failure of these magnets also affects the longitudinal emittance. For the failure of the solenoids in the SSR-A section, we observe a maximum emittance growth of $90 \%$,

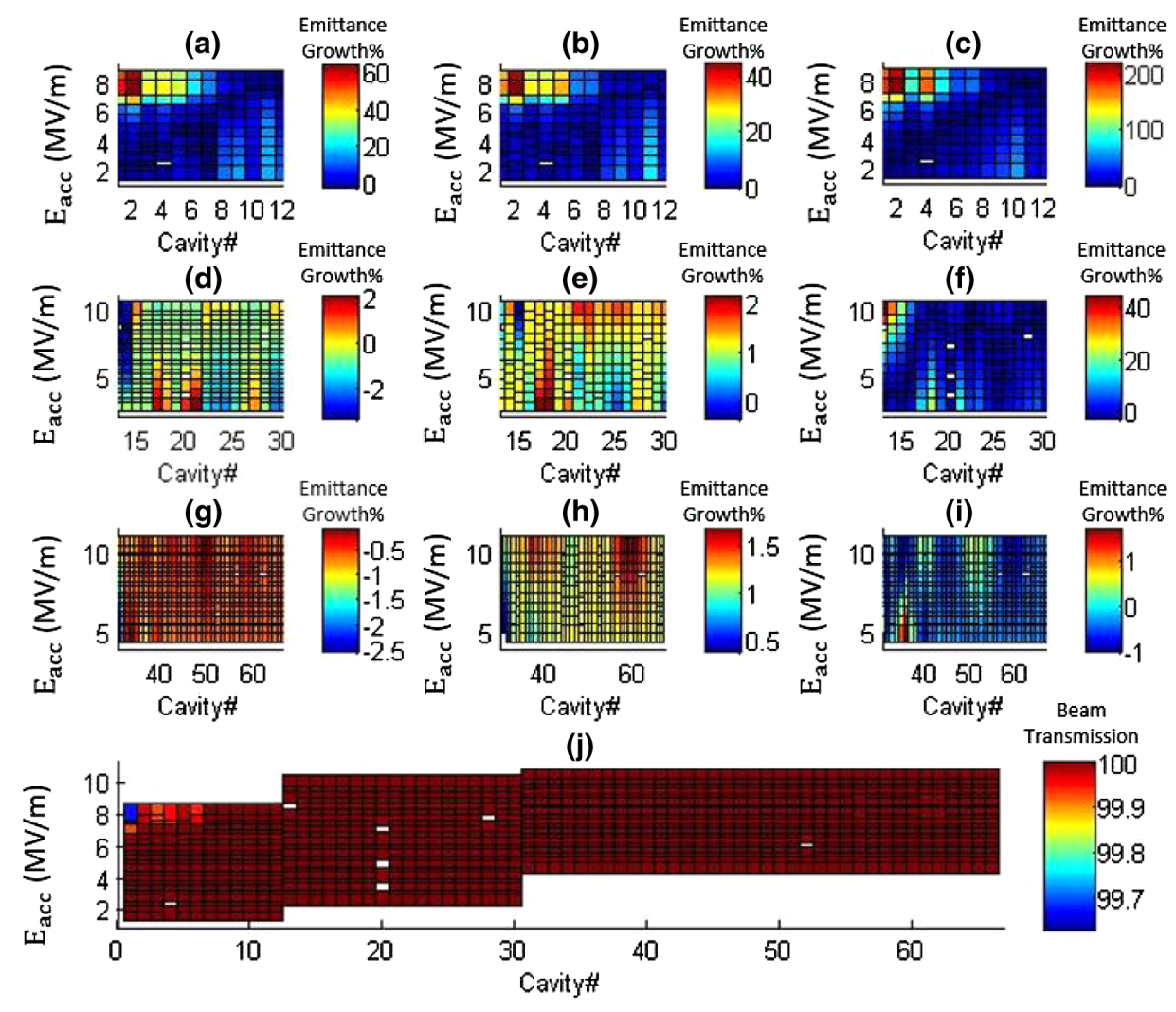

FIG. 43. (a),(b),(c) variation of X, Y, and Z emittance as a function of accelerating gradient for SSR-A; (d),(e),(f) variation of X, Y, and $\mathrm{Z}$ emittance as a function of accelerating gradient for SSR-B;(g),(h),(i) variation of X, Y, and Z emittance as a function of accelerating gradient for SSR-C; (j) beam transmission as a function of accelerating gradient for SSR-A, SSR-B, and SSR-C sections of the linac. 


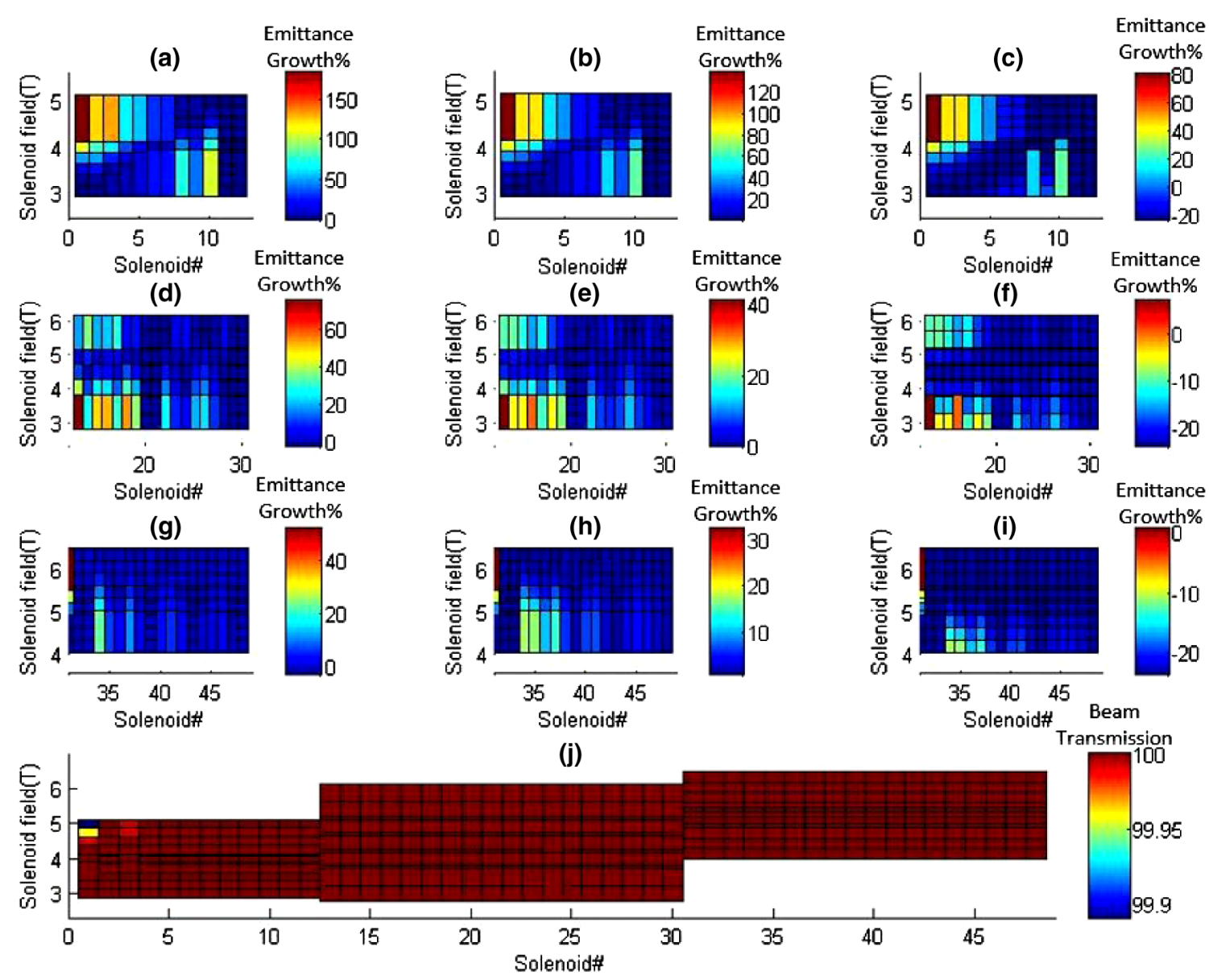

FIG. 44. (a),(b),(c) variation of X, Y, and Z emittance as a function of solenoid field for SSR-A; (d),(e),(f) variation of X, Y, and Z emittance as a function of solenoid field for SSR-B; (g),(h),(i) variation of X, Y, and Z emittance as a function of solenoid field for SSR-C; (j) beam transmission as a function of solenoid field in the SSR-A, SSR-B, and SSR-C sections of the linac.

and the growth decreases as we make the transition to the SSR-B section. The SSR-C section of the linac has the minimum effect on the longitudinal emittance with a maximum growth of $20 \%$ and a loss percentage of $2.3 \%$, due to the second solenoid's failure.

Figures 42 show that the 5th SSR-A cavity and the 12th SSR-A solenoid are most critical as their failure causes the worst emittance blow-up and beam loss. One plausible measure for compensating for their failure is through local compensation by tuning the neighboring cavity's accelerating gradient, phase, and solenoid field. An efficient approach toward such retuning is to determine the impact of the individual cavity and solenoid on the beam emittance and the particle loss and chose a cavity or a solenoid for which a minimum change in accelerating gradient or magnetic field change leads to a substantial change in beam emittance and beam transmission.

We performed a systematic study where we varied the accelerating gradient and magnetic field for each cavity and solenoid and monitored the beam emittance and beam transmission at the exit of the linac. Figure 43 shows percentage emittance growth as a function of variation in the cavity gradient of a given cavity. It is assumed that when the gradient of one cavity is varied, all the other cavities retain their nominal gradients. Figures $43(\mathrm{a}-\mathrm{c})$ show the emittance variation in $\mathrm{X}, \mathrm{Y}$, and $\mathrm{Z}$ for the cavities in SSR-A; similarly Figs. 43(d-f) for SSR-B and Figs. 43(g-i) for SSR-C. Figures 44 show the percentage emittance growth as a function of variation in the field strength of different solenoids; when one solenoid field is varied the other solenoids retain their nominal field values.

As one would expect, the failure of the spoke cavities relaxes the longitudinal focus, leading to a significant impact on the longitudinal emittance growth (rather than the transverse). Figure 43(c) shows that the maximum change in the longitudinal emittance is $213 \%$ for the second SSR-A cavity at around $8 \mathrm{MV} / \mathrm{m}$. From Fig. 43(a) it can be seen that the maximum observed transverse emittance change for the same spoke cavity is $61.6 \%$. Similar behavior is observed in the SSR-B and SSR-C sections of the linac, where the maximum longitudinal emittance growth is $41 \%$ and $1.2 \%$ for the third and the first cavities of the SSR-B and SSR-C sections respectively, and the 
TABLE XI. Beam emittance and beam loss at the exit of the linac with the failure of the critical elements and after compensation.

\begin{tabular}{|c|c|c|c|c|c|c|c|c|c|}
\hline \multirow[b]{2}{*}{$\underline{\text { Section }}$} & \multirow[b]{2}{*}{ Element } & \multicolumn{3}{|c|}{ Emittance growth after failure } & \multirow[b]{2}{*}{ Loss $\%$} & \multicolumn{3}{|c|}{ Emittance growth after compensation } & \multirow[b]{2}{*}{ Loss $\%$} \\
\hline & & $\epsilon_{x}$ & $\epsilon_{y}$ & $\epsilon_{z}$ & & $\epsilon_{x}$ & $\epsilon_{y}$ & $\epsilon_{z}$ & \\
\hline SSR-A & Cavity-5 & 18.5 & 12.9 & 229.2 & 0.52 & 15.4 & 9.2 & 40.3 & 0 \\
\hline SSR-B & Cavity-9 & 40.7 & 30.3 & 139.0 & 0.23 & 29.1 & 18.3 & 28.0 & 0 \\
\hline SSR-B & Cavity-9 & 32.0 & 16.8 & 81.9 & 0 & 26.3 & 14.7 & 20.3 & 0 \\
\hline SSR-A & Solenoid-12 & 513.0 & 434.8 & 51.8 & 3.54 & 39.3 & 35.1 & 21.5 & 0 \\
\hline SSR-B & Solenoid-9 & 323.1 & 168.8 & 27.5 & 0.51 & 28.4 & 17.4 & 13.3 & 0 \\
\hline SSR-C & Solenoid-1 & 201.3 & 55.0 & 30.0 & 0.10 & 25.3 & 13.7 & 15.8 & 0 \\
\hline
\end{tabular}

maximum transverse emittance growth is $2 \%$ and $1 \%$ for the ninth and the third cavity of SSR-B and SSR-C section respectively. As shown in Fig. 43(j), as the beam progresses in the linac, the beam energy increases, relaxing the demands on the transverse and longitudinal phase advances, and there is essentially no beam loss, rendering the later cavities in the linac less critical.

Figure 44 shows the variation in transverse emittance, longitudinal emittance, and beam transmission with the change in the solenoid fields. Clearly, solenoid failure will primarily affect the transverse emittance. We observed an enormous transverse emittance growth of $182 \%$ and a maximum longitudinal emittance growth of $45 \%$ for the first solenoid in the SSR-A section for a magnetic field of 4.6 T. The impact of these solenoids on the beam emittance decreases as the beam enters the SSR-B section of the linac. We observed a maximum transverse emittance growth of $73 \%$ and a maximum longitudinal emittance growth of $18 \%$ for the first solenoid in the SSR-B section around 3.25 T. The solenoids in the SSR-C section of the linac have minimal effect on the beam quality with a maximum transverse emittance growth of $50.5 \%$, and a maximum longitudinal emittance growth of $18.1 \%$ for the first solenoid operating at around $6 \mathrm{~T}$. Our analysis shows that within the chosen magnetic field range, only the magnetic field variation of the first solenoid of the SSR-A section causes a beam loss of $0.11 \%$ around an operating point of 4.6 T, and other solenoids from the SSR-A, SSR-B, and SSR-C sections do not cause any beam loss in the linac.

We chose the most effective cavities and solenoids obtained from the analysis shown in Figs. 43 and Figs. 44 to compensate for the beam quality deterioration and beam loss caused because of the failure of critical elements shown in Figs. 42. Table XI lists the beam emittance and beam transmission caused due to failure of critical cavities and solenoid from Figs. 42.

As shown in Table XI, with an appropriate choice of operational cavities and solenoids with an optimized accelerating gradient and magnetic field, we can significantly reduce the beam emittance and eliminate the beam loss in the linac. To compensate for the cavity failure, we chose an algorithm where we varied the accelerating field of the neighboring cavities (one at a time). After achieving an optimal value of beam emittance, we fix the accelerating gradient and move on to the next cavity until maximum compensation is obtained. The algorithm enables us to reduce the longitudinal emittance up to $188 \%$ (most critical case) for the failure of the fifth SSR-A cavity and restore full beam transmission. A similar technique was used to compensate for the solenoid failure. We varied the magnetic field of the neighboring solenoids (one at a time) and fixed the field value for an optimal beam emittance. In the case of solenoid failure, we could compensate for the transverse emittance by $474 \%$ for the failure of the first (most critical) SSR-A solenoid. As shown in Table XI, we also looked at other critical element failure and adjusted the field values to obtain maximum possible compensation while maintaining the functional requirement of the linac.

\section{ERROR ANALYSIS}

A particle accelerator is prone to numerous kinds of static and dynamic errors that may lead to issues like emittance growth, beam halo growth, and power loss on the cavity walls. We perform statistical error studies for the MEHIPA linac using the Monte Carlo method to determine the tolerances in terms of beam mismatch, beam tilt, emittance growth, beam divergence, beam energy shift, vane voltage error, voltage phase jitter, electrode longitudinal profile, the transverse radius of curvature, electrode perpendicular and parallel displacements, electrode perpendicular and parallel tilt, longitudinal electrode displacements, cavity transverse offset, cavity rotation, field amplitude, the field phase, solenoid offset, and solenoid rotation. The errors were applied in the relevant structures, and averaged over $10^{3}$ linacs where the errors were randomly generated with a Gaussian distribution over the linac. Figure 45 shows the variation of the beam emittance and particle loss percentage as a function of the steps of the error applied for the beam, solenoid and cavity displacement. Table XIII compares the emittances before and after the use of correctors for the cases demonstrated in Fig. 45. The results obtained with all other errors are listed in Table XII, where the tolerances are determined for a maximum emittance growth of $50 \%$. We also looked at the tolerances obtained for similar 

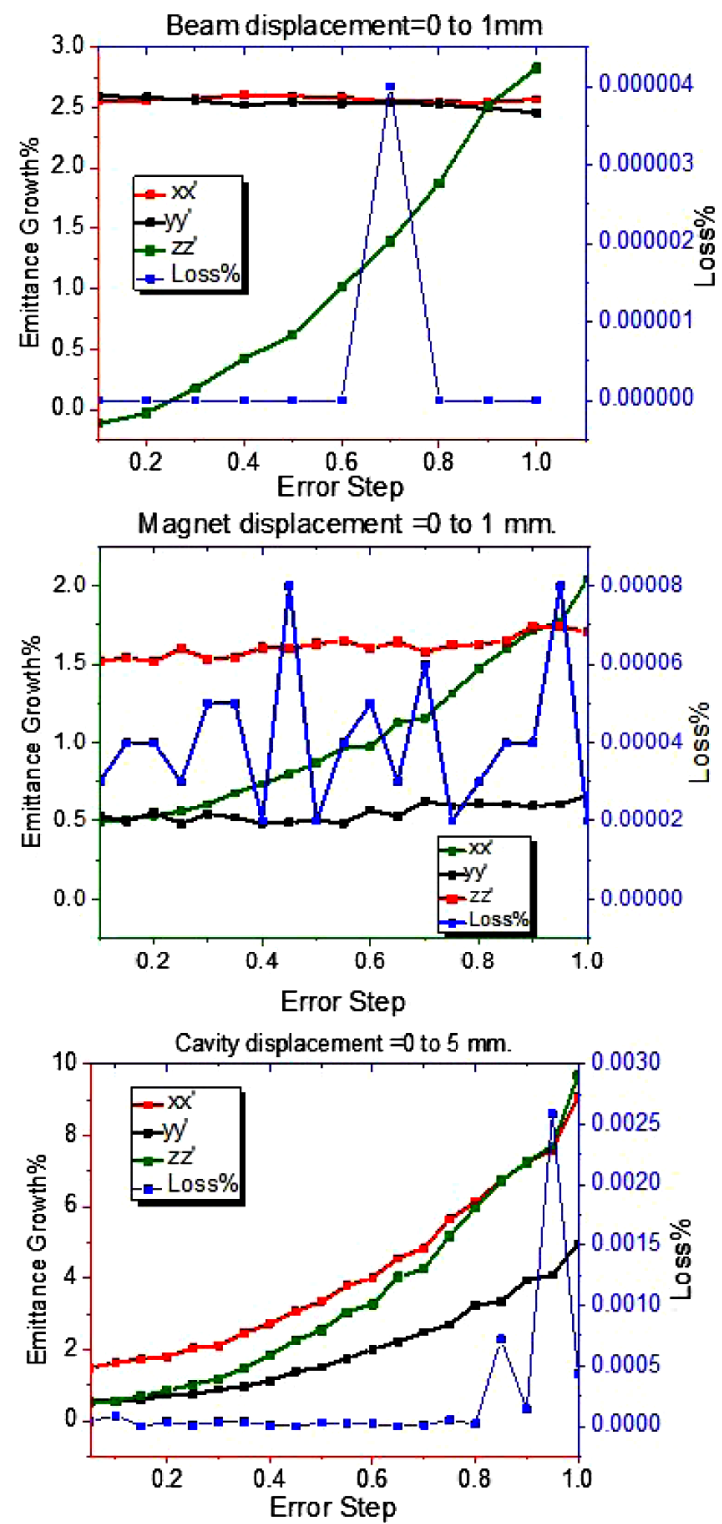

FIG. 45. Emittance growth as a function of error step for different types of error studies.

accelerator like the ESS [45] and found a close agreement with the results we obtained with our simulations.

In addition to error analysis, the knowledge of the extent to which these errors can be reduced is also significant. Here we perform studies to investigate the maximum
TABLE XII. Tolerances for MEHIPA derived from the error analysis.

\begin{tabular}{lc}
\hline \hline Parameter & Tolerance \\
\hline Beam Transverse Misalignment (mm) & 0.5 \\
Beam Divergence (mrad) & 1 \\
Transverse Emittance (\%) & 5 \\
Beam Mismatch (\%) & 15 \\
Beam Energy Shift (keV) & 10 \\
Beam Tilt about transverse axis (deg) & 0.1 \\
Vane Voltage Error (\%) & 1 \\
Voltage Phase Jitter (deg) & 1 \\
Electrode longitudinal Profile (mm) & 0.03 \\
Transverse Radius of Curvature (mm) & 0.03 \\
Electrode Perpendicular and Parallel & 0.05 \\
$\quad$ Displacement (mm) & \\
Electrode Perpendicular and Parallel Tilt (mm) & 0.04 \\
Longitudinal Electrode Displacement (mm) & 0.04 \\
Cavity Transverse Offset (mm) & 0.5 \\
Cavity Rotation about Transverse axis (deg) & 0.5 \\
Field Amplitude Error (\%) & 1 \\
Phase Jitter (deg) & 1 \\
Solenoid Transverse Offset (mm) & 0.5 \\
Solenoid Rotation about Transverse axis (deg) & 0.5 \\
Solenoid Field Error $(\%)$ & 1.5 \\
Ideal emittance at the exit of the RFQ & $0.25(\mathrm{~T})$ and \\
$\quad$ ( $\pi$ mm-mrad) & $0.33(\mathrm{~L})$ \\
Ideal emittance at the exit of the SC & $0.22(\mathrm{~T})$ and \\
$\quad$ section of the linac ( $\pi$ mm-mrad) & $0.31(\mathrm{~L})$ \\
\hline \hline
\end{tabular}

possible compensation for these errors using correctors. Table XIII shows the simulation results for the beam, cavity, and solenoid displacement errors in the linac with and without corrector for the maximum error case in Fig. 45. The solenoid fields, cavity field amplitude and rf phase of the downstream linac were tuned iteratively to minimize the emittance growth and partile loss at the exit of the linac. As Table-XIII suggests the emittance growth as well as the particle loss can be reduced significantly with the accelerating and focusing elements present in the linac.

Our analysis shows that even with the errors introduced because of RFQ and MEBT, the superconducting section of the MEHIPA linac is capable enough to deliver a beam with the required beam quality after employing the right correctors.

TABLE XIII. Emittance growth and beam loss as a function of displacement errors with and without correctors averaged over $10^{3}$ linacs.

\begin{tabular}{|c|c|c|c|c|c|}
\hline Error type & Value & $\begin{array}{c}\text { Emittance without } \\
\text { corrector(xx', yy’, zz') }\end{array}$ & $\begin{array}{l}\text { Emittance with } \\
\text { corrector(xx', yy', zz') }\end{array}$ & $\begin{array}{l}\text { Loss } \% \text { without } \\
\text { corrector }\end{array}$ & $\begin{array}{l}\text { Loss } \% \text { with } \\
\text { corrector }\end{array}$ \\
\hline Beam displacement (mm) & 2.5 & $2.5,2.5,2.8$ & $0.55,0.62,1.3$ & 0 & 0 \\
\hline Magnet displacement (mm) & 1 & $1.5,0.5,2$ & $0.28,0.37,0.97$ & $2 \times 10^{-4}$ & 0 \\
\hline Cavity displacement (mm) & 5 & $9,5,9.5$ & $6.3,2.9,5.4$ & $5 \times 10^{-4}$ & 0 \\
\hline
\end{tabular}




\section{CONCLUSION}

We have presented detailed physics design and optimization studies for the medium energy high intensity proton accelerator (MEHIPA) that is one of the few CW, high energy accelerators with such high current. The dependence of the beam quality was analyzed in terms of the space-charge nonlinearity and beam current. Our analysis shows that with an increase in space-charge nonlinearity, from $4 \mathrm{D}$ water bag to parabolic to 3 and $6 \sigma$ Gaussian, the emittance, as well as the beam halo parameter increase, and the maximum increase is observed for the Gaussian distribution. Final simulations were performed with a $6 \sigma$ Gaussian as the output from the ion source to obtain an upper limit on the emittance and halo growth. A $10 \mathrm{~mA}$, $50 \mathrm{keV}$, proton beam was matched to a $3 \mathrm{MeV}, 325 \mathrm{MHz}$ RFQ using an optimized LEBT channel and a suitable focusing scheme to avoid emittance growth and halo development in the lower energy section of the linac. The beam dynamics through a $3 \mathrm{MeV}$ RFQ was performed, and the beam quality and transmission were examined for various beam currents. The designed RFQ demonstrates an excellent performance an emittance decrease of $1.3 \%$ in the transverse plane with a transmission of $99.7 \%$. The beam from the RFQ was transported and matched to the superconducting section of MEHIPA using a MEBT channel. Two sets of quadrupole doublets were used to perform transverse matching and three two-gap buncher cavities were employed to perform longitudinal matching of the beam. The MEBT section of the linac causes an emittance growth of $3.6 \%, 1.8 \%$ and $1.5 \%$ in $x-x^{\prime}$, $\mathrm{y}-\mathrm{y}^{\prime}$, and z-z', respectively.

For the superconducting section of the MEHIPA linac, the rf design and optimization of the spoke cavities (SSR-A, SSR-B, and SSR-C) was performed using CST Microwave Studio. Spoke base radii and spoke equator major and minor radii were varied to keep the peak surface electric field below $40 \mathrm{MV} / \mathrm{m}$, the peak surface magnetic field below $70 \mathrm{mT}$, and maximize the R/Q value while keeping the resonant frequency at $325 \mathrm{MHz}$. The final optimized peak surface electric and magnetic field values for SSR-A, SSR-B, and SSR-C are $36.64 \mathrm{MV} / \mathrm{m}, 38.6 \mathrm{MV} / \mathrm{m}$, and $37.84 \mathrm{MV} / \mathrm{m}$, and $69.52 \mathrm{mT}, 68.7 \mathrm{mT}$, and $63.14 \mathrm{mT}$, respectively.

The optimized spoke cavity designs were used to perform multipacting studies assuming a Niobium (discharged clean) material. For SSR-A, the multipacting yield (MPY) varies between 1.02 to 1.14 with two barriers at $3.5 \mathrm{MV} / \mathrm{m}$ and $7 \mathrm{MV} / \mathrm{m}$. For SSR-B, the MPY is lower than SSR-A with the minimum value of 1.02 at $1 \mathrm{MV} / \mathrm{m}$ and a maximum of 1.11 at $8 \mathrm{MV} / \mathrm{m}$. SSR-B also exhibits two multipacting barriers peaking at $4 \mathrm{MV} / \mathrm{m}$ and $8 \mathrm{MV} / \mathrm{m}$, respectively. The multipacting was observed to be most severe in the case of the SSR-C cavity where the value of the MPY varies between 1 and 1.4 with three $\mathrm{MP}$ barriers at $4 \mathrm{MV} / \mathrm{m}, 6 \mathrm{MV} / \mathrm{m}$, and $9 \mathrm{MV} / \mathrm{m}$. Therefore, to reduce the MPY, a double corner radius was introduced at the end walls, leading to a significant reduction of MPY to below 1.2. We also looked at the effect of the higher-order monopole, dipole, and quadrupole modes in terms of their R/Q. For higher-order monopole modes, the $\mathrm{R} / \mathrm{Q}$ quickly damps down with increasing $\beta$ and the modes pose no threat in terms of the beam power coupling to these monopoles. The R/Q of the dipole and quadrupole modes shows that the R/Q values for these modes are less than $10^{-8}$ and, therefore, present no threat in terms of cryogenic losses and beam instabilities.

The SC lattice for the MEHIPA linac was designed using the optimized spoke cavities, and superconducting solenoids with optimized transverse and longitudinal phase advances to avoid collective beam instabilities and parametric resonances. The lattice consists of 12 SSR-A cavities housed in a single cryomodule, 18 SSR-B cavities equally distributed in two cryomodules and 36 SSR-C cavities evenly distributed in six cryomodules.

Beam dynamics simulations were performed, keeping the maximum operating gradient of SSR-A, SSR-B, and SSR-C below $8 \mathrm{MV} / \mathrm{m}, 10 \mathrm{MV} / \mathrm{m}$, and $11 \mathrm{MV} / \mathrm{m}$, respectively. With optimized transverse and longitudinal phase advances, synchronous phase, and input beam Twiss parameters, we observe an emittance growth of 9.2\%, $11.1 \%$, and $4.7 \%$ in the $x_{-}, y-$, and z-planes respectively. The halo parameter demonstrates similar behavior with an increase of $17 \%, 10 \%$, and $-8.5 \%$ in the $x^{-}, y-$, and z-directions, respectively.

The rf amplifier power requirement was estimated from the beam dynamics simulations and with a margin of $80 \%$ we obtained a total load of $18 \mathrm{~kW}, 39.6 \mathrm{~kW}$, and $90 \mathrm{~kW}$ for the SSR-A, SSR-B, and SSR-C rf amplifiers, respectively. This article also details a comprehensive reliability analysis against the failure of the accelerating and focusing elements in the linac. The criticality of individual components was determined, and retuning of most sensitive elements was performed to compensate for the beam quality deterioration in the case of cavity or solenoid failure. Our analysis shows that the element in the vicinity of inter cryomodule drift are most critical in determining the beam quality and beam transmission in the linac but, in case of failure of these elements other cavities and solenoids can be returned to regain the beam quality to a significant extent and eliminate the beam loss.

Finally, a statistical error analysis and correction scheme studies were performed for the MEHIPA linac keeping an emittance growth limit of 50\% and power loss below $1 \mathrm{~W} / \mathrm{m}$ and found that MEHIPA linac is capable of delivering a beam with required beam quality after employing right correctors.

[1] P. Singh, S. Rao, R. Pande, T. Basak, S. Roy, M. Aslam, P. Jain, S. Srivastava, R. Kumar, P. Nema et al., Accelerator development in India for ADS programme, Pramana 68, 331 (2007). 
[2] J.-L. Biarrotte, P. Pierini, D. Vandeplassche, A. Mueller, and $\mathrm{H}$. Klein, Accelerator reference design for the MYRRHA European ADS demonstrator (2010), p. 79, https:// accelconf.web.cern.ch/LINAC2010/papers/tup020.pdf.

[3] T. Mukaiyama, Omega programme in Japan and ADS development at JAERI, in Accelerator Driven Transmutation Technologies and Applications (Proc. 2nd Int. Conf., Prague, 1998) (Citeseer, Prague, Czech Republic, 1999), pp. 1-5.

[4] J. Tang, P. Cheng, H. Geng, Z. Guo, Z. Li, C. Meng, H. Ouyang, S. Pei, B. Sun, J. Sun et al., Conceptual physics design for the China-ADS linac, Proceedings of PAC2013, Pasadena, CA USA (2013), pp. 1397-1399, https:// accelconf.web.cern.ch/pac2013/papers/thpsm04.pdf.

[5] C. Park, H. Ahn, B. Choi, T. Eom, Y. Cho, J. Han, J. Lee, W. Park, S. Kang, W. Song et al., The KOMAC project: accelerator and transmutation project in Korea, in $K E K$ Proceedings (National Laboratory for High Energy Physics, Tsukuba, Japan, 1998), pp. 319-322.

[6] I. A. D. D. BARC, Design of a $200 \mathrm{MeV}$ medium energy high intensity proton accelerator for the Indian ADS programme, External Report BARC/2016/e/016, 2016.

[7] J. V. Mathew, S. Rao, R. Pande, and P. Singh, Beam emittance measurements and simulations of injector line for radio frequency quadrupole, Rev. Sci. Instrum. 86, 073306 (2015).

[8] R. Pande, P. Singh, S. Rao, S. Roy, and S. Krishnagopal, Optimization of solenoid based low energy beam transport line for high current $\mathrm{H}^{+}$beams, J. Instrum. 10, P02001 (2015).

[9] S. Roy, S. Rao, R. Pande, S. Krishnagopal, and P. Singh, Electromagnetic and beam dynamics studies of a high current drift-tube linac for LEHIPA, J. Instrum. 9, P06007 (2014).

[10] M. Ball, A. Burov, B. Chase, A. Chakravarty, A. Chen, S. Dixon, J. Edelen, A. Grassellino, D. Johnson, S. Holmes et al., The PIP-II conceptual design report, Tech. Rep. (Argonne National Lab.(ANL), Argonne, IL (United States); Fermi National, 2017).

[11] E. D. Courant and H. S. Snyder, Theory of the alternatinggradient synchrotron, Ann. Phys. (N.Y.) 281, 360 (2000).

[12] C. Allen and T. Wangler, Beam halo definitions based upon moments of the particle distribution, Phys. Rev. Accel. Beams 5, 124202 (2002).

[13] D. Uriot and N. Pichoff, Status of TraceWIN code, Proceedings of the IPAC'15 (2015), pp. 92-94, http:// www.jacow.org/IPAC2015/papers/MOPWA008.pdf.

[14] N. Chauvin, O. Delferrière, R. Duperrier, R. Gobin, P. Nghiem, and D. Uriot, Transport of intense ion beams and space charge compensation issues in low energy beam lines, Rev. Sci. Instrum. 83, 02B320 (2012).

[15] J. Potter, S. Williams, and F. Humphry, and G. Rodenz, Radio frequency quadrupole accelerating structure research at Los Alamos, IEEE Trans. Nucl. Sci. 26, 3745 (1979).

[16] O. Buneman, Dissipation of currents in ionized media, Phys. Rev. 115, 503 (1959).

[17] R. Duperrier, Toutatis: A radio frequency quadrupole code, Phys. Rev. Accel. Beams 3, 124201 (2000).
[18] P. Berrutti, I. Gonin, T. Khabiboulline, M. Parise, D. Passarelli, G. Romanov, F. Ruiu, A. Sukhanov, and V. Yakovlev, New design of SSR2 spoke cavity for the PIP-II SRF linac, in 19th Int. Conf. on RF Superconductivity (SRF'19), Dresden, Germany, 30 June-05 July 2019 (JACOW Publishing, Geneva, Switzerland, 2019), pp. 600-604.

[19] H. J. Cha, J. Yoon, S. W. Jang, K.-R. Kim, S. H. Park, and E.-S. Kim, Design of a balloon-shaped superconducting single spoke resonator, J. Korean Phys. Soc. 75, 117 (2019).

[20] M. O. Hyun, Y. W. Jo, H. Jung, and Y. Kim, Mechanical design of single spoke resonator type-2 (SSR2) superconducting cavity for risp, in 14th Int. Conf. on Heavy Ion Accelerator Technology (HIAT'18), Lanzhou, China, 2226 October 2018 (JACOW Publishing, Geneva, Switzerland, 2019), pp. 125-128.

[21] Y. Wang, H. Liu, J. Dai, P. Sha, Z. Deng, B. Li, M. Fan, A. Li, P. Qu, X. Wu et al., RF design of a compact $648 \mathrm{MHz}$ single spoke cavity at CSNS, Radiat. Detection Technol. Methods 3, 56 (2019).

[22] J. Tamura, K. Hasegawa, E. Kako, Y. Kondo, T. Konomi, F. Maekawa, S.-i. Meigo, H. Sakai, K. Umemori, and B. YeeRendón, Electromagnetic design of the prototype spoke cavity for the JAEA ADS linac, in 19th Int. Conf. on RF Superconductivity (SRF'19), Dresden, Germany, 30 June05 July 2019 (JACOW Publishing, Geneva, Switzerland, 2019), pp. 399-402.

[23] Z. Meng, H. Feisi, and P. Weimin, Design and selection of $325 \mathrm{MHz}$ medium $\beta$ spoke cavity, Intense Laser Part. Beam 31, 31115101 (2019).

[24] A. Sukhanov, C. Contreras-Martinez, F. Garcia, B. Hanna, S. Kazakov, Y. Pischalnikov, O. Prokofiev, S. Samani, W. Schappert, I. Terechkine et al., Characterization of SSR1 cavities for PIP-II linac, in 19th Int. Conf. on RF Superconductivity (SRF'19), Dresden, Germany, 30 June-05 July 2019 (JACOW Publishing, Geneva, Switzerland, 2019), pp. 1120-1123.

[25] G. Olry, J.-L. Biarrotte, S. Blivet, S. Bousson, C. Joly, T. Junquera, J. Lesrel, A. Mueller, H. Saugnac, P. Szott et al., Development of spoke cavities for the eurisol and eurotrans projects, Physica C (Amsterdam) 441, 201 (2006).

[26] G. Olry, J. Biarrotte, S. Blivet, S. Bousson, F. Chatelet, T. Junquera, A. Le Goff, J. Lesrel, C. Miélot, A. Mueller et al., Development of SRF spoke cavities for low and intermediate energy ion linacs, in Proceedings of the 11th Workshop on RF Superconductivity, Lübeck/Travemünder, Germany, Vol. 3 (2003) p. 76, http://accelconf.web.cern.ch/ accelconf/SRF2003/papers/tup55.pdf.

[27] L. Ristori, Y. Pischalnikov, G. Apollinari, T. Khabiboulline, J. Ozelis, D. Sergatskov, E. Borissov, I. Gonin, R. Webber, R. Wagner et al., Design, fabrication and testing of single spoke resonators at Fermilab, Fermi National Accelerator Lab.(FNAL), Batavia, IL (United States) Report No. FERMILAB-PUB-09-437-TD, 2009.

[28] I. Gonin, T. Khabiboulline, L. Ristori, A. Sergatskov, R. Webber, R. Wagner, A. Mukherjee, J. Ozelis, and G. Lanfranco, High gradient test of the HINS SSR1 single spoke resonator, Technical Report, 2008. 
[29] L. Han, S. Peng, D. Jian-Ping, H. Hong, and W. Qun-Yao, Design study on very low beta spoke cavity for China-ads, Chin. Phys. C 36, 761 (2012).

[30] P. Duchesne, S. Bousson, G. Duthil, G. Olry, D. Reynet, and S. Molloy, Design of the $352 \mathrm{MHz}$, beta 0.50 , doublespoke cavity for ESS, Proceedings of SRF2013, Paris, France (2013), https://accelconf.web.cern.ch/SRF2013/ papers/frioc01.pdf.

[31] P. Berrutti, M. Awida, I. Gonin, J. Ostiguy, N. Solyak, A. Vostrikov, and V. Yakovlev, Optimization of the geometric beta for the SSR2 cavities of Project X, in Proceedings of the 3rd International Particle Accelerator Conference, New Orleans, LA, 2012 (IEEE, Piscataway, NJ, 2012).

[32] H. Padamsee, J. Knobloch, T. Hays et al., RF Superconductivity for Accelerators (Wiley Online Library, New York, 2008), Vol. 2011.

[33] J. Biarrotte, G. Olry, and D. Uriot, A 90-160/180 MeV Spoke linac as an option for the CERN Linac4/SPL, Technical Report, 2006.

[34] M. Guan, X. Wang, L. Ma, Y. Zhou, H. Zhao, C. Xin, L. Yang, W. Wu, and X. Yang, Magnetic field and strain measurements of a superconducting solenoid magnet for c-ads injector-ii during excitation and quench test, J. Supercond. Novel Magn. 26, 2361 (2013).

[35] M. Guan, X. Wang, L. Ma, Y. Zhou, H. Zhao, C. Xin, L. Yang, W. Wu, and X. Yang, Magnetic field and strain measurements of a superconducting solenoid magnet for cADS injector-II during excitation and quench test, J. Supercond. Novel Magn. 26, 2361 (2013).

[36] C. M. Studio, CST Microwave studio suite (Version 2014), Darmstadt, Germany (2002), http://cst-academy.com/ images/Facilitator_manual_10-09-2006_revised_.pdf.

[37] T. P. Wangler, RF Linear accelerators (John Wiley \& Sons, New York, 2008).
[38] P. Berrutti, M. Avida, I. Gonin, J. Ostiguy, N. Solyak, A. Vostrikow, and V. Yokavlev, Optimization of the geometric beta for the SSR2 cavities of Project X, in Proceedings of the 3rd International Particle Accelerator Conference, New Orleans, LA, 2012 (IEEE, Piscataway, NJ, 2012).

[39] S.-h. Kim, M. Doleans, D.-o. Jeon, and R. Sundelin, Higher-order-mode (HOM) power in elliptical superconducting cavities for intense pulsed proton accelerators, Nucl. Instrum. Methods Phys. Res., Sect. A 492, 1 (2002).

[40] P. Berutti, T. Khabiboulline, and G. Romanov, Multipactor discharge in the PIP-II superconducting spoke resonators, Fermi National Accelerator Lab.(FNAL), Batavia, IL (United States), Technical division, Technical Report No. TD-16-005, 2016.

[41] J. Struckmeier and M. Reiser, Theoretical studies of envelope oscillations and instabilities of mismatched intense charged particle beams in periodic focusing channels, Part. Accel. 14, 227 (1983).

[42] I. Hofmann, Stability of anisotropic beams with space charge, Phys. Rev. E 57, 4713 (1998).

[43] P. Berrutti, M. Awida, B. Shteynas, I. Gonin, N. Solyak, V. Yakovlev, and A. Saini, Effects of the rf field asymmetry in sc cavities of Project $\mathrm{X}$, in Proceedings of the $3 \mathrm{rd}$ International Particle Accelerator Conference, New Orleans, LA, 2012 (IEEE, Piscataway, NJ, 2012), WEPPC047.

[44] L. Groening, I. Hofmann, W. Barth, W. Bayer, G. Clemente, L. Dahl, P. Forck, P. Gerhard, M. Kaiser, M. Maier et al., Experimental Evidence of Space Charge Driven Emittance Coupling in High Intensity Linear Accelerators, Phys. Rev. Lett. 103, 224801 (2009).

[45] M. Eshraqi, R. De Prisco, R. Miyamoto, E. Sargsyan, and H. Thomsen, Statistical error studies in the ESS linac, in Proceedings of the 5th International Particle Accelerator Conference, THPME044 (Dresden, Germany, 2014), http://accelconf.web.cern.ch/AccelConf/IPAC2014/papers/ thpme044.pdf. 